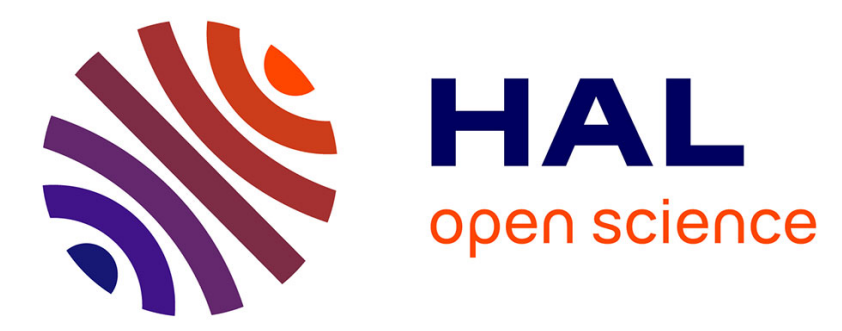

\title{
Astrocyte-derived Jagged-1 mitigates deleterious Notch signaling in amyotrophic lateral sclerosis
}

\author{
Annelies Nonneman, Nathan Criem, Sebastian Lewandowski, Rik Nuyts, \\ Dietmar Thal, Frank Pfrieger, John Ravits, Philip van Damme, An Zwijsen, \\ Ludo van den Bosch, et al.
}

\section{To cite this version:}

Annelies Nonneman, Nathan Criem, Sebastian Lewandowski, Rik Nuyts, Dietmar Thal, et al.. Astrocyte-derived Jagged-1 mitigates deleterious Notch signaling in amyotrophic lateral sclerosis. Neurobiology of Disease, 2018, 119, pp.26-40. 10.1016/j.nbd.2018.07.012 . hal-02408650

\section{HAL Id: hal-02408650 https://hal.science/hal-02408650}

Submitted on 13 Dec 2021

HAL is a multi-disciplinary open access archive for the deposit and dissemination of scientific research documents, whether they are published or not. The documents may come from teaching and research institutions in France or abroad, or from public or private research centers.
L'archive ouverte pluridisciplinaire HAL, est destinée au dépôt et à la diffusion de documents scientifiques de niveau recherche, publiés ou non, émanant des établissements d'enseignement et de recherche français ou étrangers, des laboratoires publics ou privés. 


\title{
Astrocyte-derived Jagged-1 mitigates deleterious Notch signaling in amyotrophic lateral sclerosis
}

\author{
Nonneman Annelies ${ }^{\mathrm{a}, \mathrm{b}}$, Criem Nathan ${ }^{\mathrm{b}, \mathrm{c}, \mathrm{d}}$, Lewandowski Sebastian A. ${ }^{\mathrm{e}, \mathrm{f}}$, Nuyts Rik ${ }^{\mathrm{a}, \mathrm{b}}$, \\ Thal Dietmar R. ${ }^{\mathrm{g}, \mathrm{h}}$, Pfrieger Frank W. ${ }^{\mathrm{i}}$, Ravits John ${ }^{\mathrm{j}}$, Van Damme Philip ${ }^{\mathrm{a}, \mathrm{b}, \mathrm{h}}$, Zwijsen An ${ }^{\mathrm{b}, \mathrm{c}, \mathrm{d}}$, \\ Van Den Bosch Ludo ${ }^{\mathrm{a}, \mathrm{b}}$, Robberecht Wim ${ }^{\mathrm{a}, \mathrm{b}, \mathrm{h}, *}$ \\ ${ }^{a}$ KU Leuven - University of Leuven, Department of Neurosciences, Laboratory of Neurobiology and Experimental Neurology, and Leuven Brain Institute (LBI), Herestraat \\ 49, B-3000 Leuven, Belgium \\ ${ }^{\mathrm{b}}$ VIB, Center for Brain \& Disease Research, Herestraat 49, B-3000 Leuven, Belgium \\ ${ }^{c}$ KU Leuven - University of Leuven, Department of Cardiovascular Sciences, Center for Molecular and Vascular Biology, Herestraat 49, B-3000 Leuven, Belgium \\ ${ }^{\mathrm{d}} \mathrm{KU}$ Leuven - University of Leuven, Department of Human Genetics, Herestraat 49, B-3000 Leuven, Belgium

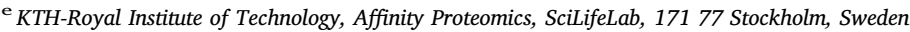 \\ ${ }^{\mathrm{f}}$ Karolinska Institute, Department of Clinical Neuroscience, 17177 Stockholm, Sweden \\ ${ }^{g}$ KU Leuven - University of Leuven, Department of Neurosciences, Laboratory for Neuropathology, Herestraat 49, B-3000 Leuven, Belgium \\ ${ }^{\mathrm{h}}$ University Hospitals Leuven, Department of Neurology, Herestraat 49, B-3000 Leuven, Belgium \\ ${ }^{i}$ Institute of Cellular and Integrative Neurosciences, CNRS UPR 3212, University of Strasbourg, 67084 Strasbourg, France \\ ${ }^{\mathrm{j}}$ University of California, Department of Neurosciences, 9500 Gilman Drive, La Jolla, San Diego, CA 92093-0624, USA
}

\section{A R T I C L E I N F O}

\section{Keywords:}

Amyotrophic lateral sclerosis

Notch

Jagged-1

Cis

Trans

Astrocyte

Motor neuron

Neurodegeneration

Conditional knock-out mice

Genetic modulation

\begin{abstract}
A B S T R A C T
Amyotrophic lateral sclerosis (ALS) is a late-onset devastating degenerative disease mainly affecting motor neurons. Motor neuron degeneration is accompanied and aggravated by oligodendroglial pathology and the presence of reactive astrocytes and microglia. We studied the role of the Notch signaling pathway in ALS, as it is implicated in several processes that may contribute to this disease, including axonal retraction, microgliosis, astrocytosis, oligodendrocyte precursor cell proliferation and differentiation, and cell death. We observed abnormal activation of the Notch signaling pathway in the spinal cord of SOD ${ }^{G 93 A}$ mice, a well-established model for ALS, as well as in the spinal cord of patients with sporadic ALS (sALS). This increased activation was particularly evident in reactive GFAP-positive astrocytes. In addition, one of the main Notch ligands, Jagged-1, was ectopically expressed in reactive astrocytes in spinal cord from ALS mice and patients, but absent in resting astrocytes. Astrocyte-specific inactivation of Jagged-1 in presymptomatic SOD $1^{G 93 A}$ mice further exacerbated the activation of the Notch signaling pathway and aggravated the course of the disease in these animals without affecting disease onset. These data suggest that aberrant Notch signaling activation contributes to the pathogenesis of ALS, both in sALS patients and SOD $1^{G 93 A}$ mice, and that it is mitigated in part by the upregulation of astrocytic Jagged-1.
\end{abstract}

\section{Introduction}

Amyotrophic lateral sclerosis (ALS) is a late-onset, rapidly progressing degenerative disease mainly affecting motor neurons. Patients present muscle denervation, weakness and atrophy of muscles and ultimately paralysis. Denervation of the respiratory muscles leads to respiratory failure and death, which typically occurs 3 to 5 years after disease onset (Swinnen \& Robberecht, 2014; Brown \& Al-Chalabi, 2017).

Approximately $10 \%$ of patients have an affected relative and suffer from the hereditary form of ALS. This "familial ALS" can be caused by mutations in a variety of genes, of which hexanucleotide repeat expansions in chromosome 9 open reading frame 72 (C9ORF72) and mutations in superoxide dismutase 1 (SOD1), in fused in sarcoma (FUS) and TAR DNA-binding protein 43 (TDP-43) are the most prevalent ones (Swinnen \& Robberecht, 2014; Brown \& Al-Chalabi, 2017; Renton et al., 2014). The remaining $90 \%$ of patients have no known affected family members and are classified as having "sporadic ALS" (sALS). Nevertheless, causal mutations can be identified in about $10 \%$ of these sALS patients indicating a hereditary form of ALS (Swinnen \& Robberecht,

\footnotetext{
* Corresponding author at: Laboratory of Neurobiology, KU Leuven, Campus Gasthuisberg, O\&N4 Herestraat 49, Box 602, B-3000 Leuven, Belgium.

E-mail address: Wim.Robberecht@kuleuven.be (W. Robberecht).
} 
2014; Brown \& Al-Chalabi, 2017).

Transgenic mice overexpressing human mutant SOD1 have been used extensively to study ALS (Van Damme et al., 2017). This mouse model nicely recapitulates human ALS pathology with adult-onset and progressive motor neuron degeneration and muscle atrophy, ultimately evolving into paralysis and death (Gurney, 1994). Studies using this mouse model have shown that motor neuron degeneration in ALS is a non-cell autonomous process with an important contribution of neighboring glial cells (Boillee et al., 2006; Philips \& Robberecht, 2011; Ilieva et al., 2009). Mutant SOD1-containing reactive microglia and astrocytes are toxic to motor neurons, although the molecular mechanism of this toxicity is incompletely understood (Boillee et al., 2006; Philips \& Robberecht, 2011; Yamanaka et al., 2008; Haidet-Phillips et al., 2011; Appel et al., 2011; Henkel et al., 2009; Hall et al., 1998). Oligodendrocytes also contribute actively to the pathogenesis of ALS (Kang et al., 2013; Philips et al., 2013; Lee et al., 2012; Nonneman et al., 2014). Their extensive degeneration is accompanied by marked proliferation of oligodendrocyte precursor cells to compensate for the loss. However, the differentiation and functional maturation of these new cells is incomplete, resulting in defective myelination of axons and deficient metabolic support of neurons (Nonneman et al., 2014; Morrison et al., 2013; Nave, 2010). Thus, these findings demonstrate that the interaction between motor neurons and glial cells is pivotal in the process of degeneration (Philips \& Robberecht, 2011). It is generally accepted that glial cells modify the course of the degenerative process, of which the onset is determined by the motor neurons.

A critical mediators of cell-cell interactions is the Notch signaling pathway (Artavanis-Tsakonas et al., 1999; Lai, 2004). This pathway converts an extracellular signal received from a ligand-carrying neighboring cell into gene expression changes in the nucleus of the Notch receptor-carrying cell. In mammals, four transmembrane Notch receptors (NOTCH-1, -2, -3, 4), five transmembrane canonical ligands (JAGGED-1, -2; DELTA-LIKE-1, -3, 4) and several non-canonical ligands have been identified. Upon ligand binding, the Notch receptor is cleaved by $\alpha$ - and $\gamma$-secretases, resulting in the release of the Notch intracellular domain (NICD), which serves as the active signaling fragment. NICD translocates to the nucleus, where it forms a transcription-activation complex with the cofactors 'recombination signal binding protein for immunoglobulin kappa J region' (RBPjk) and 'mastermind-like proteins' (MAML-1, -2, - 3), and initiates the transcription of target genes, such as the HES genes (HES-1, -5, -7) and the $H E Y$ genes (HEY-1, -2, -L) (Kopan \& Ilagan, 2009; Ilagan \& Kopan, 2007; Bray, 2006). However, when a cell carries both the Notch receptor and the ligand, then this ligand impedes the activation of the receptor, a process called cis-inhibition (del Alamo et al., 2011).

Notch signaling has been extensively studied as a developmental pathway regulating cell-cell communication and controlling cell-fate specification by inhibiting cell differentiation and promoting proliferation (Artavanis-Tsakonas et al., 1999; Ilagan \& Kopan, 2007). The study of Notch signaling in the adult organism was hampered for a long time because loss of Notch signaling causes severe embryonic phenotypes and early lethality (Louvi \& Artavanis-Tsakonas, 2006; Hamada et al., 1999; Huppert et al., 2000; Oka et al., 1995; Yoon \& Gaiano, 2005). The availability of inducible Cre-loxP targeting technology makes it possible to choose the timing and tissue type in which Notch signaling is modulated and allows to investigate the role of Notch signaling in adulthood and in adult-onset disease models (Sato et al., 2012; Ables et al., 2011).

The Notch signaling pathway is implicated in several processes that are relevant to the pathogenesis of ALS, such as axonal outgrowth and retraction (Bonini et al., 2013; Berezovska et al., 1999; Redmond et al., 2000; Sestan et al., 1999; El Bejjani \& Hammarlund, 2012), neuromuscular junction maturation (de Bivort et al., 2009), microgliosis (Jurynczyk \& Selmaj, 2010; Grandbarbe et al., 2007; Wei et al., 2011; Arumugam et al., 2006), astrocytosis (Marumo et al., 2013; Shimada et al., 2011; Zhang et al., 2015; Kamei et al., 2012), proliferation and differentiation of oligodendrocyte precursor cells into mature oligodendrocytes (Wang et al., 1998; Genoud et al., 2002; Popko, 2003; Park \& Appel, 2003), and cell death (Yang et al., 2004; Arumugam et al., 2011). In addition, abnormal Notch signaling has been linked to neurodegenerative diseases (Mathieu et al., 2013) such as Alzheimer's disease (Woo et al., 2009; Berezovska et al., 1998), multiple sclerosis (Jurynczyk \& Selmaj, 2010; Aparicio et al., 2013; John et al., 2002), spinal muscular atrophy (Caraballo-Miralles et al., 2013), prion disease (Ishikura et al., 2005; Dearmond \& Bajsarowicz, 2010) and ischemic stroke (Arumugam et al., 2006). Animal models for ALS revealed contradictory results about the role of Notch. In Drosophila, one study showed that loss-of-function mutations of Notch or of downstream target genes extended the lifespan of TDP-43 transgenic flies (Zhan et al., 2013), whereas another study showed that increased Notch expression suppressed the toxicity induced by the GR dipeptide repeats associated with C9orf72 expansions (Yang et al., 2015). In ALS mouse models, no functional Notch studies are available. Only increased expression of Notch-3 and the downstream target gene Hes- 1 has been reported in the gastrocnemius muscle of the mutant SOD1 mouse (von Grabowiecki et al., 2015), but the role of Notch signaling in ALS pathogenesis remains undefined.

Therefore, this study aimed to investigated Notch signaling in ALS patient samples and in the mutant SOD $1^{G 93 A}$ mouse model for ALS, and to explore whether interfering with this pathway affects disease in the mouse model. The latter was achieved using inducible Cre-loxP targeting technology. Our findings demonstrate overactivation of the Notch signaling pathway in the lumbar spinal cord of both sALS patients and the SOD $1^{G 93 A}$ mouse model compared to controls and demonstrate overexpression of the ligand Jagged-1 in reactive astrocytes of ALS spinal cord. Astrocyte-specific inactivation of Jagged-1 further exacerbated the activation of the Notch signaling pathway and accelerated disease progression in $S O D 1^{G 93 A}$ mice without affecting disease onset. Together, these data suggest that overactivation of the Notch signaling pathway contributes to the pathogenesis of ALS, both in sALS patients and SOD $1^{G 93 A}$ mice, and that it is mitigated in part by the upregulation of Jagged-1 in reactive astrocytes.

\section{Materials \& methods}

\subsection{Mice}

Mice overexpressing human mutant SOD1 (SOD1 ${ }^{G 93 A}$, B6.Cg-Tg (SOD1*G93A)1Gur/J, JAX stock number 004435) and human wildtype SOD1 (SOD1 ${ }^{W T}$, B6.Cg-Tg(SOD1)2Gur/J, JAX stock number 002298), control non-transgenic mice (C57BL/6J, JAX stock number 000664), Jagged-1 $1^{\text {lox } / \text { lox }}$ mice (B6;129S-Jag1 $1^{\text {tm2Grid }} / \mathrm{J}$, JAX stock number 010618), Notch-1 ${ }^{\text {lox } / l o x}$ mice (B6.129X1-Notch $1^{\text {tm2Rko }} /$ GridJ, JAX stock number 007181), Rosa- ${ }^{\text {lox }}$ STOP ${ }^{l o x}$-YFP mice (B6.129X1-Gt(ROSA) $26 \operatorname{Sor}^{\text {tm1(EYFP)Cos }} / \mathrm{J}$, JAX stock number 006148) and CAGG-CreER mice (B6.Cg-Tg(CAG-cre/Esr1*)5Amc/J, JAX stock number 004682) were obtained from The Jackson Laboratory (Bar Harbor, Maine, USA). Cx30-CreER mice (Slezak et al., 2007; Barnabe-Heider et al., 2010) were kindly provided by Frank W. Pfrieger (INCI, Strasbourg, France). Cremediated recombination was induced at the age of 60 days by administering tamoxifen as described before (Philips et al., 2013). Maintenance of mice used in this study and all experiments were carried out in strict accordance with protocols approved by the Ethical Committee of the University of Leuven, Belgium (P149/2011 and P281/2015).

\subsection{Human samples}

The expression data set of the whole-genome exon splicing array provided by the group of John Ravits (Rabin et al., 2010) on motor neurons and the remaining anterior horn is publically available (www. ncbi.nlm.nih.gov/geo, accession number GSE18920). Motor neurons were isolated by Laser Capture Microdissection (LCM) from lumbar 
spinal cords of 12 sporadic ALS patients (sALS) and 10 control individuals. The GeneChip raw data CEL-files were reanalyzed using the Affymetrix Expression Console to obtain summarized expression values for transcripts presented as normalized probe intensity units.

Human formalin-fixed paraffin-embedded lumbar spinal cord samples used for histology were kindly provided by one of the authors (John Ravits). All human lumbar spinal cord samples from 6 patients diagnosed with ALS using revised El Escorial criteria (4 males, ages 41-74 years, disease duration of 1.5-4.75 years; 2 females, ages 58-74 years, disease duration of 3-5.75 years) and from 6 control persons with no pathological evidence of neurological disease (5 males, ages 38-82 years; 1 females, age 63 years) were collected at the University of California San Diego using informed consents and protocols approved by the Human Research Protection Program of the University of California San Diego. Postmortem time intervals ranged from 3 to $8.5 \mathrm{~h}$ (mean $=5.3 \mathrm{~h}$ ).

\subsection{Mouse tissue collection and sample preparation}

Mice were sacrificed using an overdose of Nembutal $(10 \mathrm{mg} / \mathrm{kg}$; Ceva chemicals, Hornsby, NSW, Australia). For mRNA and protein experiments, lumbar spinal cords were collected, flash-frozen in liquid nitrogen and maintained at $-80^{\circ} \mathrm{C}$ until further use. For immunohistochemical staining experiments, mice were transcardially perfused with PBS $1 \times$ (Sigma-Aldrich, St. Louis, USA) followed by perfusion with $4 \%$ paraformaldehyde (PFA, Sigma-Aldrich, St. Louis, USA). Subsequently, lumbar spinal cord tissue was dissected, post-fixed with $4 \%$ PFA for $2 \mathrm{~h}$ on ice, dehydrated overnight in a $30 \%$ sucrose solution at $4{ }^{\circ} \mathrm{C}$, embedded in Optimal Cutting Temperature (O.C.T.) compound mounting medium for cryotomy (VWR, Radnor, PA, USA) and stored at $-80^{\circ} \mathrm{C}$ until further processing.

\subsection{Quantitative real-time polymerase chain reaction}

Total RNA was isolated from mouse lumbar spinal cords using the Tripure isolation reagent (Roche, Mannheim, Germany) according to the manufacturer's instructions. For cDNA synthesis $1 \mu \mathrm{g}$ of total RNA was reverse transcribed using SuperScript ${ }^{\circledast}$ III First-Strand Synthesis Systme kit (Invitrogen, Carlsbad, CA, USA) with oligo-dT and random hexamer primers. Subsequently, quantitative PCR (qPCR) was performed in a volume of $10 \mu \mathrm{l}$ using Taqman Fast Universal PCR Master Mix $2 \times$ (Applied Biosystems, Carlsbad, CA, USA), TaqMan gene expression $20 \times$ assays (Applied Biosystems, Carlsbad, CA, USA) and $4 \mu 1$ of a 1/15 dilution of cDNA template. Following TaqMan gene expression $20 \times$ assays with FAM dye (Thermo Fisher Scientific, Waltham, MA, USA) were used: Notch-1 (Mm00627185_m1), Notch-2 (Mm00803077_m1), Notch-3 (Mm01345646_m1), Notch-4 (Mm00440525_m1), Jagged-1 (Mm00496902_m1, for standard gene expression analysis), Jagged-1 (Mm00496904_m1, for recombination efficiency analysis), Jagged-2 (Mm01325629_m1), Dll-1 (Mm01279269_m1), Dll-3 (Mm00432854_m1), Dll-4 (Mm00444619_m1), F3/Contactin-1 (Mm00514374_m1), NB3/ Contactin-6 (Mm00516899_m1), Rbpjk (Mm00770450_m1), Maml-1 (Mm00614627_m1), Maml-2 (Mm00620617_m1), Maml-3 (Mm01294189_m1), Hes-1 (Mm01342805_m1), Hes-5 (Mm00439311_g1), Hes-7 (Mm00473576_m1), Hey-1 (Mm00468865_m1), Hey-2 (Mm00469280_m1), Hey-L (Mm00516555_m1), Hprt (Mm00446968_m1), Gapdh (Mm99999915_g1) and Por2a (Mm00839502_m1). Thermal cycling was performed on a $\mathrm{ViiA}^{\mathrm{TM}} 7$ Real-Time PCR System (Applied Biosystems, Carlsbad, CA, USA). All samples were run in triplicate. Reference genes used for normalization were determined by geNorm analysis. Biogazelle qBase +2.6 Software was used for raw data analysis, quality control and quantification of calibrated normalized relative gene expression. Scaling was done to the average of the control group.

\subsection{Western blot}

Proteins were extracted from mouse lumbar spinal cords as described before (Philips et al., 2013). Forty micrograms of protein lysates together with Pierce ${ }^{\mathrm{TM}}$ lane marker reducing sample buffer $5 \times$ (Thermo Fisher Scientific, Waltham, MA, USA) were loaded on a 7.5\% (for large proteins) or a $12 \%$ (for small proteins) fresh Sodium Dodecyl sulphate polyacrylamide (SDS-PAGE) gel and processed as described previously (Beel et al., 2017). Following primary antibodies diluted in Tris Buffered Saline with $0.1 \%$ Tween-20 (TBST) were used: rabbit antiNOTCH-1 (Cell Signaling Technology, D1E11, RRID: AB_2153354, 1:1000 in TBST with 5\% BSA, overnight at $4{ }^{\circ} \mathrm{C}$ ), rabbit anti-NOTCH-2 (Cell Signaling Technology, D76A6, RRID: AB_10693319, 1:1000 in TBST with 5\% BSA, $2 \mathrm{~h}$ at room temperature (RT)), rabbit antiJAGGED-1 (Cell Signaling Technology, 28H8, RRID: AB_10693295, 1:1000 in TBST with $5 \%$ BSA, overnight at $4{ }^{\circ} \mathrm{C}$ ), rabbit anti-RBPjk (Cell Signaling Technology, D10A4, RRID: AB_2665555, 1:1000 in TBST, $1 \mathrm{~h}$ at RT), rabbit anti-Cleaved NOTCH-1 (Val1744) (Cell Signaling Technology, D3B8, RRID: AB_2153348, 1:1000 in TBST with 5\% BSA, overnight at $4{ }^{\circ} \mathrm{C}$ ), mouse anti-GAPDH (Ambion, AM4300, RRID: AB 437392, 1:5000 in TBST, $1 \mathrm{~h}$ at RT) and mouse anti-alpha TUBULIN (Sigma-Aldrich, T6199, RRID: AB_477583, 1:5000 in TBST, $1 \mathrm{~h}$ at RT). Protein bands were visualized using SuperSignal ${ }^{\mathrm{TM}}$ West Femto Maximum Sensitivity ECL Substrate (for NOTCH-1, NOTCH-2, JAGGED-1, RBPjk and Cleaved NOTCH-1 (NICD-1); Thermo Fisher Scientific, Waltham, MA, USA) or Pierce ${ }^{\mathrm{TM}}$ ECL Western Blotting Substrate (for GAPDH and alpha-TUBULIN; Thermo Fisher Scientific, Waltham, MA, USA). Protein levels were normalized to GAPDH and alpha-TUBULIN.

\subsection{Immunohistochemistry}

Twenty micrometer-thick cryosections were cut from O.C.T. embedded mouse lumbar spinal cords. Immunofluorescent stainings were performed as described previously (Philips et al., 2013), except for the JAGGED-1 and the NICD-1 staining. Following primary antibodies were used: goat anti-JAGGED-1 (Santa Cruz Biotechnology, C-20 sc-6011, RRID: AB_649689, 1:50, overnight at $4{ }^{\circ} \mathrm{C}$ ), mouse anti-NeuN (Millipore, MAB377, RRID: AB_2298772, 1:200, $1 \mathrm{~h}$ at RT), mouse anti-GFAP (Sigma-Aldrich, G3893, RRID: AB_477010, 1:200, $1 \mathrm{~h}$ at RT), rabbit anti-IBA-1 (Wako Chemicals, 019-19741, RRID: AB_839504, 1:200, $1 \mathrm{~h}$ at RT) and goat anti-GFP (Rockland, 600-101-215M, RRID: AB_ 2612804, 1:200, $1 \mathrm{~h}$ at RT).

For the Jagged-1 staining, the standard staining protocol (Philips et al., 2013) was preceded by a pre-treatment with $0.5 \%$ sodium dodecyl surfate (Sigma-Aldrich, Saint-Louis, MO, USA) in PBS for maximal $5 \mathrm{~min}$. The specificity of the goat anti-JAGGED-1 (Santa Cruz Biotechnology, C-20, 1:50) was evaluated by overnight pre-incubation with $6 \mu \mathrm{g}$ of following JAGGED-1 neutralizing peptides: sc-6011 P blocking peptide (Santa Cruz Biotechnology, Dallas, Texas, USA) or Jagged-1 recombinant protein antigen NBP1-90208PEP (Novus Biologicals, Abingdon, England), respectively. Sections were mounted using ProLong ${ }^{\circledR}$ Gold Antifade Mountant with DAPI (Thermo Fisher Scientific, Waltham, MA, USA).

For the NICD-1 staining, sections were permeabilized with PBST and immersed in Sodium-Citrate buffer $(10 \mathrm{mM}$ Sodium Citrate, $\mathrm{pH}$, $0.01 \%$ Tween 20 ) for $30 \mathrm{~min}$ at $96^{\circ} \mathrm{C}$ followed by $20 \mathrm{~min}$ cooldown at $4{ }^{\circ} \mathrm{C}$ for antigen retrieval. Endogenous peroxidases were inactivated with $3 \% \mathrm{H}_{2} \mathrm{O}_{2}$ in methanol (30 min, at RT). After blocking for $1 \mathrm{~h}$ with $3 \%$ BSA in TBS $(150 \mathrm{mM}$ Tris $\mathrm{pH} 7.5,150 \mathrm{mM} \mathrm{NaCl})$, sections were incubated with rabbit anti-NICD-1 (Val1744) (Cell Signaling Technology, D3B8, RRID: AB_2153348, 1:100 in TBS with $0.1 \%$ Tween 20 and $3 \%$ BSA, overnight at $4{ }^{\circ} \mathrm{C}$ ). After incubation with biotinylated donkey anti-rabbit secondary antibody (Jackson ImmunoResearch 711-065-152, West Grove, PA, USA) for $1 \mathrm{~h}$ at RT, the signal intensity was amplified using the TSA biotin signal amplification detection kit (NEL700A001KT, Perkin Elmer, Waltham, MA, USA) 
according to the manufacturer's recommendations and visualized with streptavidin-Alexa-488 (Jackson ImmunoResearch 016-540-084, West Grove, PA, USA). Sections were mounted with MOWIOL (SigmaAldrich, Saint-Louis, MO, USA) supplemented with DAPI.

Thionin staining was used to visualize (motor) neurons, as described before (Beel et al., 2017). The area of the neuronal cell bodies and the ventral horn was measured and the number of motor neurons was normalized to the area of the ventral horn.

For the human JAGGED-1 staining on formalin-fixed paraffin embedded lumbar spinal cord samples from.

sALS patients and from control persons, sections $(6 \mu \mathrm{m})$ were deparaffinized and rehydrated by subsequent immersion in xylene (2 times, 10 min; VWR, Radnor, PA, USA), 100\% ethanol ( 2 times, 1 min), $95 \%$ ethanol ( $1 \mathrm{~min}), 70 \%$ ethanol $(2 \mathrm{~min}), 50 \%$ ethanol $(1 \mathrm{~min})$ and $\mathrm{H} 2 \mathrm{O}$ (5 min). Antigen retrieval was performed with sodium-citrate buffer (10 mM sodium citrate, $\mathrm{pH} 6,0.01 \%$ Tween 20$)$ supplemented with $0.1 \%$ sodium dodecyl surfate (Sigma-Aldrich, Saint-Louis, MO, USA) for $40 \mathrm{~min}$ at $96^{\circ} \mathrm{C}$ followed by $20 \mathrm{~min}$ cooldown at $4{ }^{\circ} \mathrm{C}$. Endogenous peroxidases were inactivated by $30 \mathrm{~min}$ incubation in 3\% $\mathrm{H} 2 \mathrm{O} 2$ in PBS supplemented with 50\% methanol. Blocking with $10 \%$ NDS in PBST for $1 \mathrm{~h}$ at RT, was followed by incubation with goat antiJAGGED-1 (Santa Cruz Biotechnology, C-20 sc-6011, RRID: AB_649689, $1: 50$ in PBST with $10 \%$ NDS, overnight at $4^{\circ} \mathrm{C}$ ) and with biotinylated donkey anti-goat secondary antibody (Jackson ImmunoResearch 705067-003, 1:300 in PBST, $1 \mathrm{~h}$ at RT). Subsequently, sections were incubated with streptavidine-HRP (Perkin Elmer TSA kit, NEL700A001KT, 1:100 in PBST, $30 \mathrm{~min}$ ) according to the manufacturer's instruction. After incubation with Tris- $\mathrm{HCl}(50 \mathrm{mM}$ Tris, $\mathrm{pH} 7.5$; 2 times, $5 \mathrm{~min}$ ), staining was revealed using 3,3'-diaminobenzidine (DAB, SK-4100, Vector Laboratories, Burlingame, CA) as a substrate. Sections were counterstained with Harris's haematoxilin (VWR, Radnor, PA, USA; 159-380-025), dehydrated and mounted.

For the NICD-1 staining on human samples, the sections were deparaffinized and rehydrated as described above. The NICD-1 staining procedure was performed as described for the NICD-1 staining on mouse cryosections with the following differences to the protocol: antigen retrieval was performed with TE buffer ( $10 \mathrm{mM}$ Trizma base, 1 mM EDTA, pH 9, 0.005\% Tween20) and the tyramide amplification was followed by incubation with streptavidine-HRP (Perkin Elmer TSA kit, NEL700A001KT, 1:100 in TBS with $0.1 \%$ Tween 20 and 3\% BSA, $30 \mathrm{~min}$ ). The staining was revealed using $3,3^{\prime}$-diaminobenzidine (DAB, SK- 4100, Vector Laboratories, Burlingame, CA) as a substrate and the sections were dehydrated and mounted. For proper visualization of NICD-1, no counterstaining with Harris's haematoxilin was performed in combination with the NICD-1 staining.

The human GFAP staining was performed on the BOND RX stainer (Leica Biosystems, Wetzlar, Germany) with the Bond ${ }^{\mathrm{TM}}$ Polymer Refine Detection Protocol (Leica Biosystems Wetzlar, Germany; DS9800) according to the manufacturer's instructions. Following primary antibody was used: FLEX Polyclonal Rabbit Anti-Glial Fibrillary Acidic Protein (Dako, Ready-To-Use Autostainer solution). Afterwards, sections were mounted in the Leica CV5030 Fully Automated Glass Coverslipper (Leica Biosystems Wetzlar, Germany).

Fluorescent images were taken on an Axio Imager.M1 microscope equipped with an AxioCam MRc5 camera or an AxioCam MRm camera (Zeiss, Jena, Germany). Confocal images were taken on a Leica TCS SP8 confocal laser scanning microscope (Leica Microsystems, Wetzlar, Germany). Bright-field images were taken on a Leica DM2000 LED microscope equipped with a DFC7000 digital camera (Leica Microsystems, Wetzlar, Germany). Several images (5 to 10) of the ventral horn region were taken per sample and image analysis and quantification was performed in a blinded manner using freeware ImageJ and AxioVision SE64 software.

\subsection{Evaluation of disease progression in mice: motor performance,} innervation, body weight, disease onset and endpoint determination

Motor performance was assessed twice a week from the age of 60 days onwards by the hanging grid test and the fixed rotarod performance test at $15 \mathrm{rpm}$, as previously described (Staats et al., 2016). Innervation was assessed by using a ultra pro S100 EMG monitoring setup (Natus Neurology, Pleasanton, CA, USA) with Synergy software (Acertys, Aartselaar, Belgium) on mice anesthetized with 3\% isoflurane, as described before (d'Ydewalle et al., 2011). Compound muscle action potential (CMAP) amplitudes were normalized to the CMAP amplitude measured at the age of 50 days, because the CMAP amplitudes of SOD $1^{\text {G93A }}$ mice and Jagged-1 ${ }^{\text {lox/lox }}$ CX 30 -CreER SOD $1^{\text {G93A }}$ mice measured at 50 days of age (this is before the tamoxifen-induced inactivation of Jagged-1) were not identical. Body weight was measured twice a week starting from the age of 60 days as a generalized parameter indicating disease progression. Disease onset was defined the day when a mouse was not able to hold on for $60 \mathrm{~s}$ on the hanging grid test anymore. End stage was determined as the time point when paralyzed mice could no longer turn themselves within $10 \mathrm{~s}$ when placed on their side. At this moment mice were sacrificed. Survival was defined the time from birth to end stage. Disease duration was defined as the period between disease onset and end stage.

\subsection{Statistical analysis}

GraphPad Prism 5 software (Graphad Software, La Jolla, CA, USA) was used for all statistical analysis. Unpaired $t$-test, Mann-Whitney $U$ test, one- and two-way ANOVA with post hoc analysis and Log-rank Mantel-Cox test were performed as specified in the figure legends. Normality was evaluated with Shapiro-Wilk normality test. Quantitative data are presented as mean \pm SEM. P-values smaller than 0.05 were considered statistically significant.

\section{Results}

3.1. Increased expression of key mediators of Notch signaling in the lumbar spinal cord of $S O D 1^{G 93 A}$ mice

We first examined the expression (mRNA and protein) of the Notch receptors (Notch-1, -2, -3 and -4), the ligands (Jagged-1 and -2; Deltalike-1, -3 and -4 ) and the transcription cofactors (RBPjk and Mastermind-like-1, -2 and - 3) of the canonical Notch signaling pathway in the lumbar spinal cord of the SOD $1^{G 93 A}$ mouse. In addition, two noncanonical ligands (F3/Contactin-1 and NB3/Contactin-6) were studied for completeness. Lumbar spinal cords of age-matched non-transgenic mice and of age-matched $S O D 1^{W T}$ mice were used as controls.

Quantitative PCR showed that the mRNA expression of the Notch receptors increases with disease progression (Fig. 1A). Until day 60, the mRNA expression levels of all four Notch receptors were unaltered compared to age-matched control SOD ${ }^{W T}$ mice and non-transgenic mice. However, during disease (125 days and 150 days), a significant upregulation of all Notch receptors in the affected lumbar spinal cord was observed, whereas their expression in control SOD $1^{W T}$ spinal cords remained unchanged (Fig. 1A).

Similarly, the mRNA expression of the canonical Notch ligand Jagged-1 was increased significantly in $S O D 1^{G 93 A}$ lumbar spinal cord. Interestingly, this increase was already observed at the age of 60 days, well before disease onset and did not further increase with disease progression (Fig. 1B). The expression of other canonical and non-canonical ligands was not significantly changed (Supplementary Fig. 1).

We next studied the expression of these factors at the protein level. We focused on the proteins NOTCH-1 and NOTCH-2, because their mRNA increase was most prominent during disease progression. In agreement with the mRNA data, Western blot analysis showed a significant increase of the levels of the processed forms of the receptor 
A

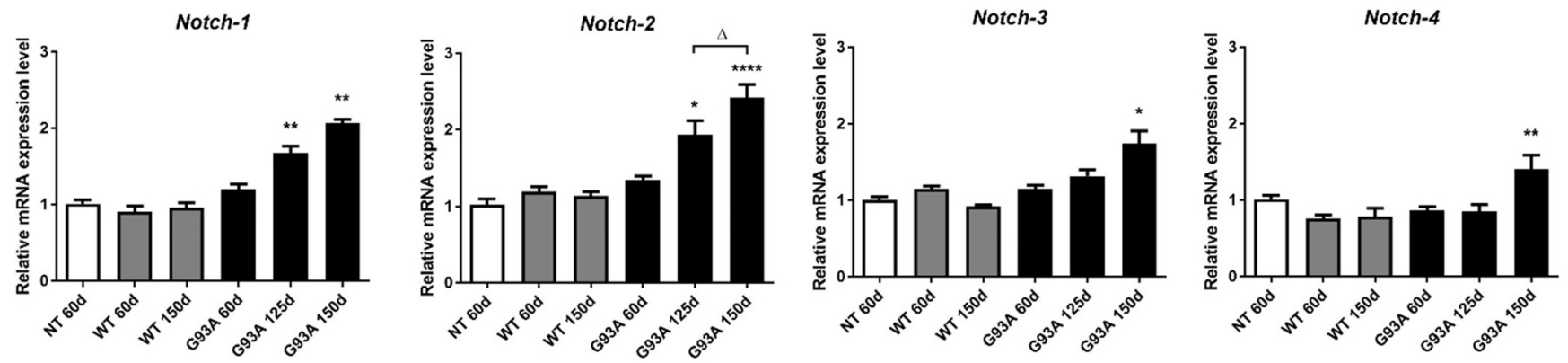

B

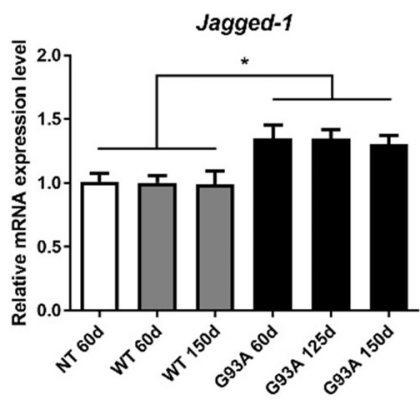

D

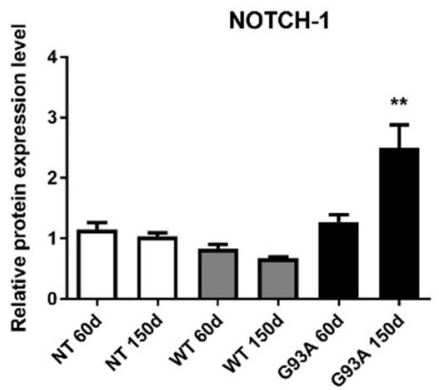

C

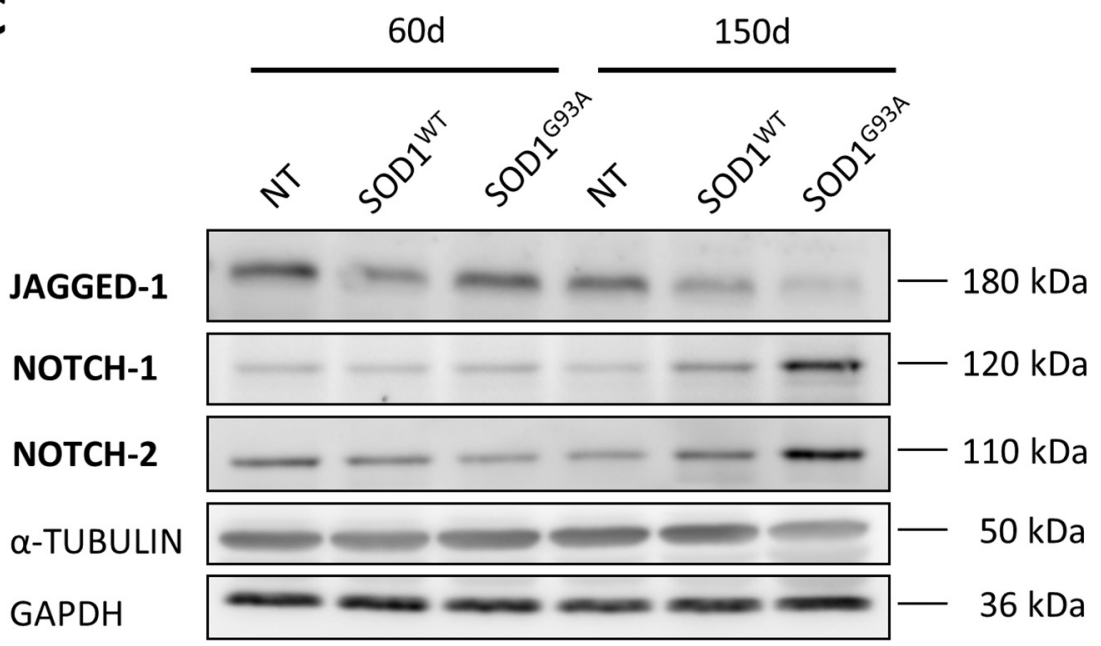

E

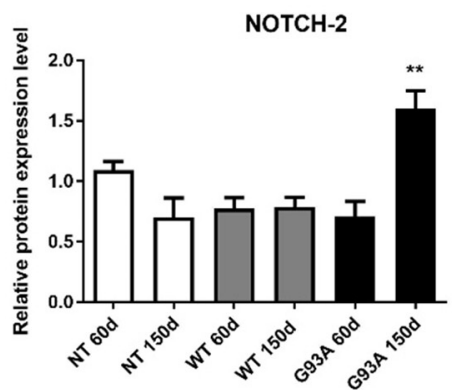

$\mathbf{F}$

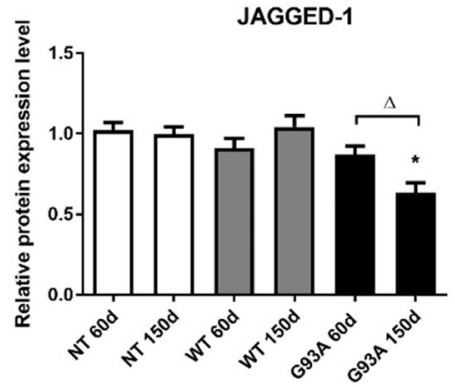

Fig. 1. The Notch receptors and the ligand Jagged-1 are more abundantly expressed in the affected lumbar spinal cord of SOD $1^{\text {G93A }}$ mice compared to control mice. mRNA levels of (A) the Notch receptors and (B) the ligand Jagged-1 in the affected lumbar spinal cord of SOD1 ${ }^{\text {G93A }}$ mice (G93A) at different disease stages $\left(60 \mathrm{~d}=\right.$ asymptomatic stage; $125 \mathrm{~d}=$ symptomatic stage; $150 \mathrm{~d}=$ end stage), determined by qRT-PCR. Lumbar spinal cords of age-matched SOD1 ${ }^{W T}$ mice (WT) and non-transgenic mice (NT) were used as controls. Expression data are normalized to Hprt and Gapdh and scaled to NT 60d. (C) Western Blot analysis showing NOTCH1, NOTCH-2 and JAGGED-1 in the affected lumbar spinal cord of SOD ${ }^{\mathrm{G} 93 \mathrm{~A}}$ mice (G93A) at different disease stages $(60 \mathrm{~d}=$ asymptomatic stage; $150 \mathrm{~d}=$ end stage). Lumbar spinal cords of age-matched SOD1 ${ }^{\mathrm{WT}}$ mice (WT) and non-transgenic mice (NT) were used as controls. $\alpha$-TUBULIN and GAPDH were used for loading controls. (D, E and F) Protein levels of indicated proteins determined by densitometric analysis of the Western Blots shown in (C). One-way ANOVA with Tukey's posthoc analysis: ${ }^{*} \mathrm{p}<0.05,{ }^{* *} \mathrm{p}<0.01,{ }^{* * *} \mathrm{p}<0.0001$ with all other groups, except when indicated differently, and $\Delta \mathrm{p}=0.06$ indicates a tendency, $\mathrm{n}=6$ mice per group for qRT-PCR and $\mathrm{n}=3$ mice per group for western Blot. Data represent mean \pm SEM.

proteins NOTCH-1 (molecular weight of $120 \mathrm{kDa}$, Fig. 1C-D) and NOTCH-2 (molecular weight of $110 \mathrm{kDa}$, Fig. 1C and E) in lumbar spinal cords of end stage $S O D 1^{G 93 A}$ mice ( 150 days) compared to asymptomatic SOD1 ${ }^{G 93 A}$ mice (60 days) and age-matched control $S O D 1^{W T}$ or non-transgenic mice. In contrast to its increased mRNA levels, the protein expression of full-length JAGGED-1 (molecular weight of $180 \mathrm{kDa}$ ) was significantly reduced in the spinal cord from end stage $S O D 1^{G 93 A}$ mice compared to asymptomatic $S O D 1^{G 93 A}$ mice (60 days), and compared to age-matched control $S O D 1^{W T}$ or nontransgenic mice (Fig. 1C and F), indicating increased JAGGED-1 
A
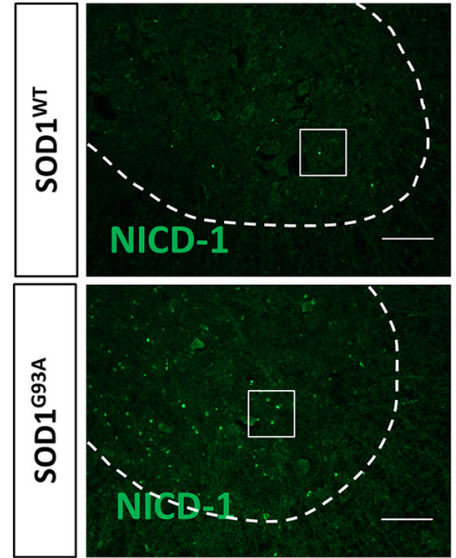

B

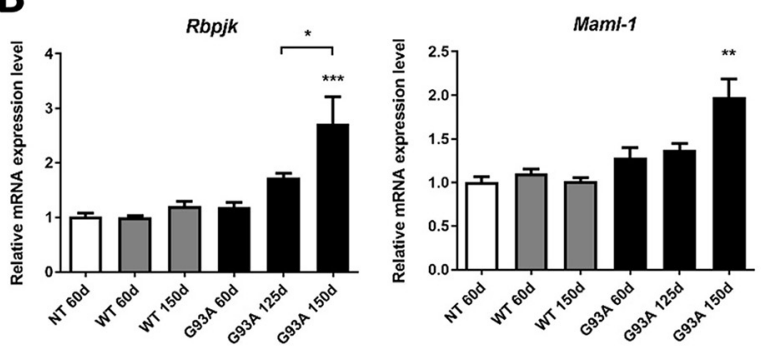

C

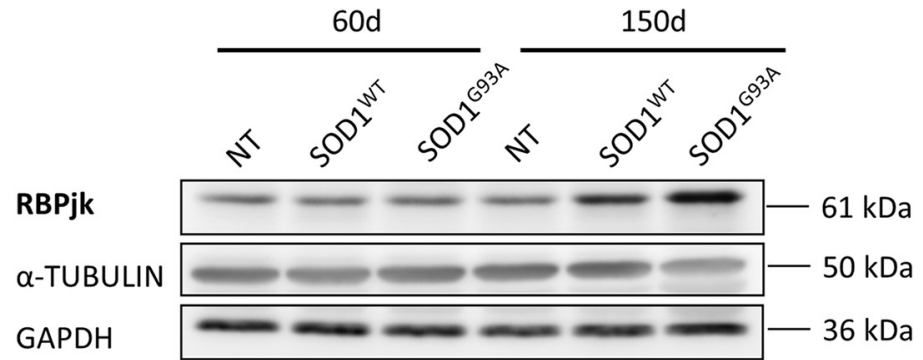

E

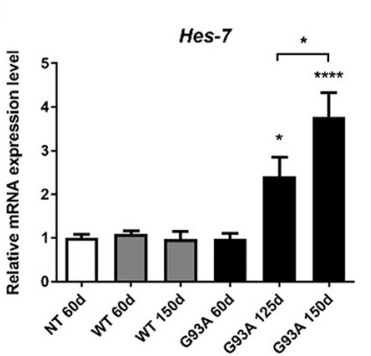

$\mathbf{G}$

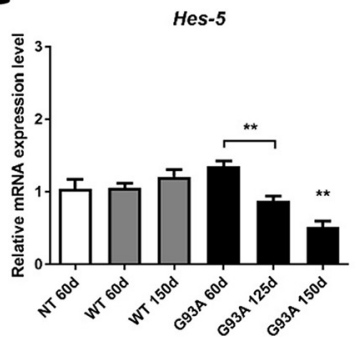

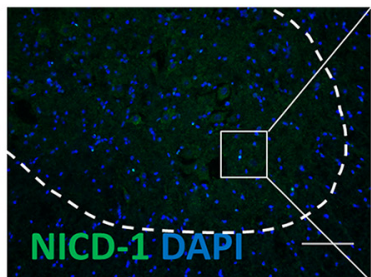
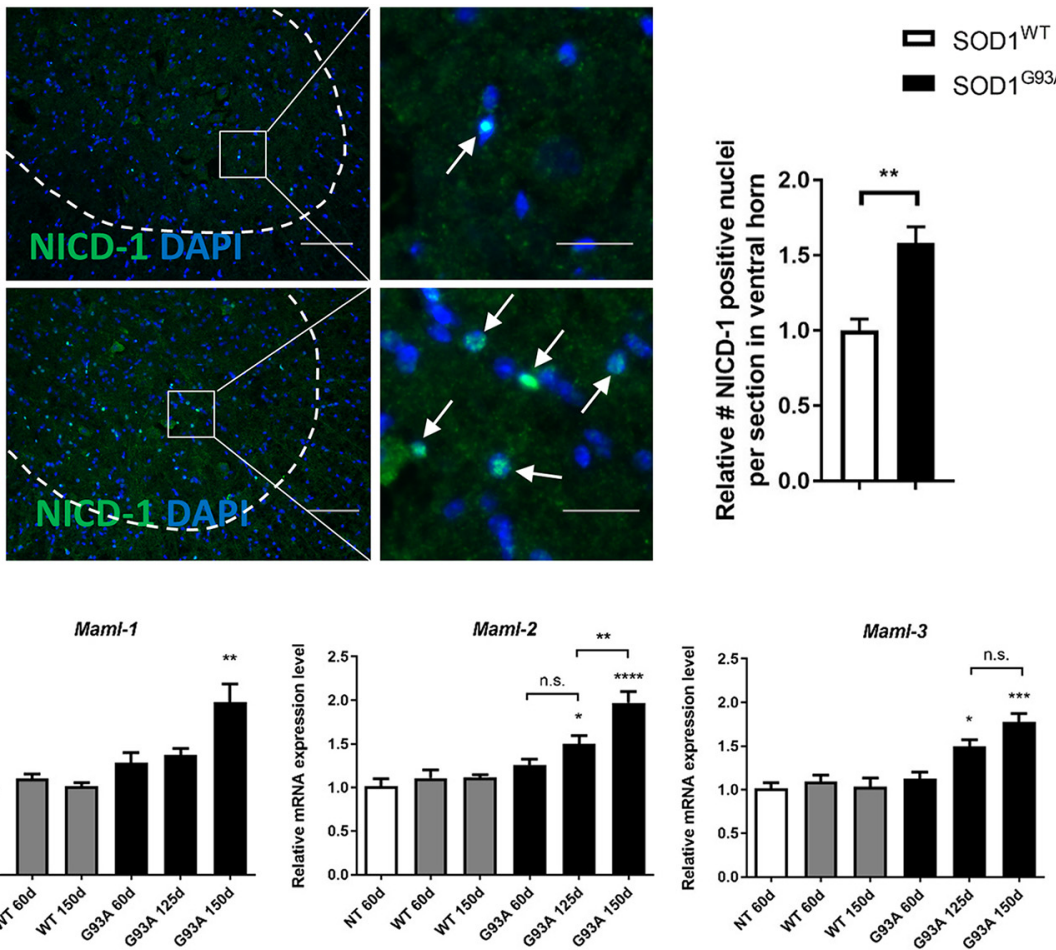

D

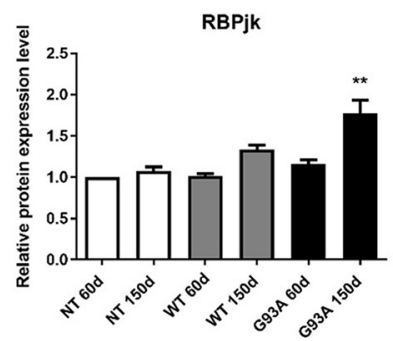

$\mathbf{F}$
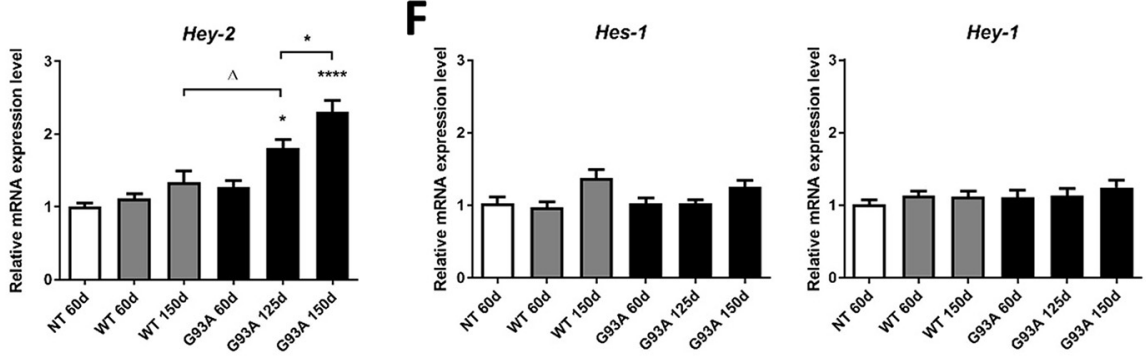

processing (cleavage, endocytosis and degradation) after signal transduction (D'Souza et al., 2008; Hansson et al., 2010; He et al., 2014; Lavoie \& Selkoe, 2003; Le Bras et al., 2011). (caption on next page)

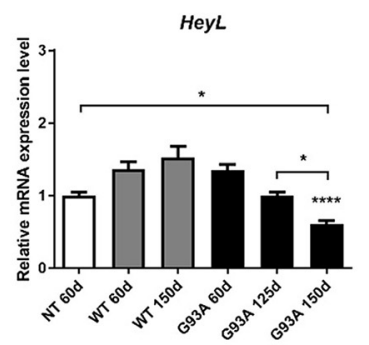

3.2. Increased activation of the Notch signaling pathway in lumbar spinal cord of SOD $1^{\text {G93A }}$ mice

The mRNA and protein expression profiles described above suggest that the Notch signaling pathway is aberrantly activated in the spinal 


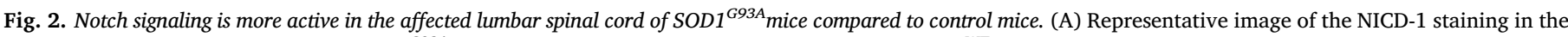

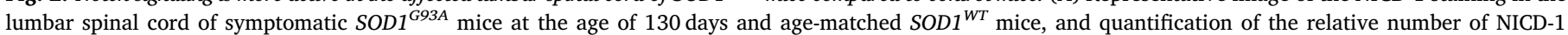

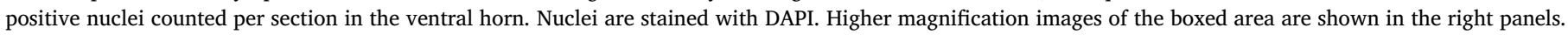

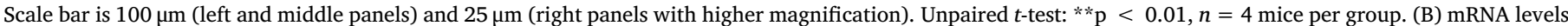

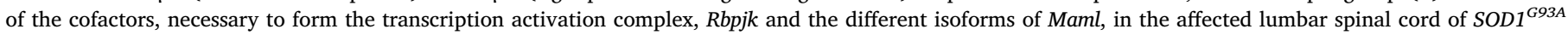

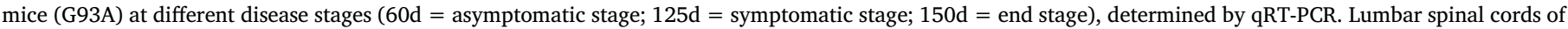

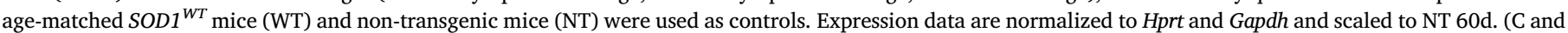

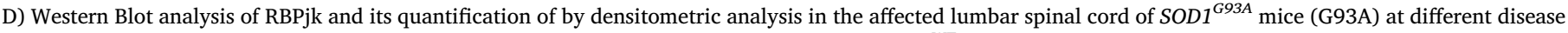

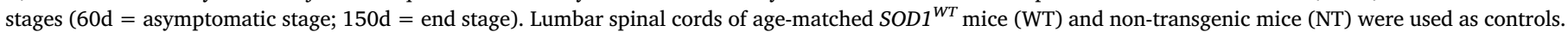

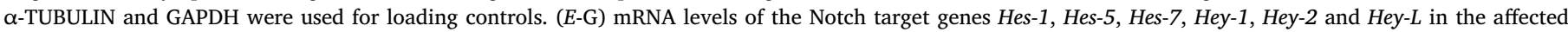

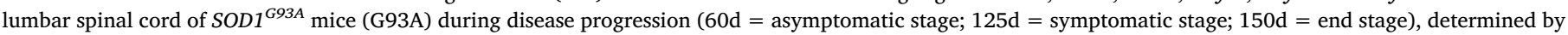

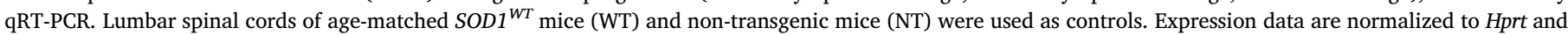

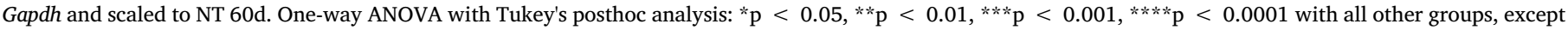

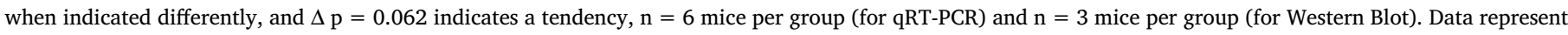
mean \pm SEM.
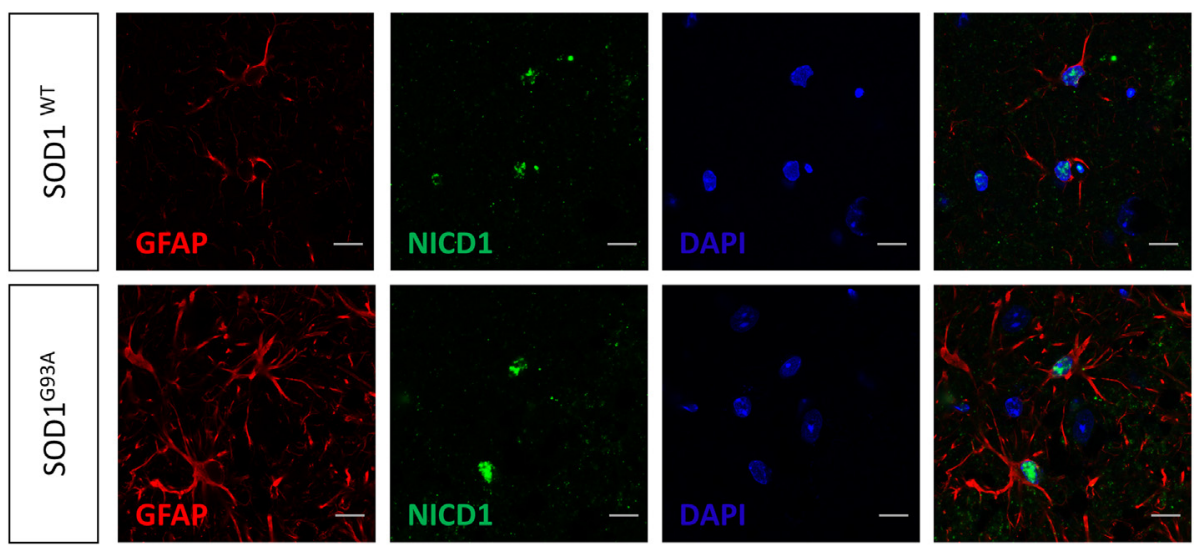

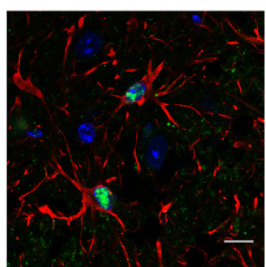

Fig. 3. Astrocytes mediate Notch signaling in the affected lumbar spinal cord of SOD1 ${ }^{G 93 A}$ mice. Immunofluorescent staining of NICD-1 in the ventral horn of the lumbar spinal cord from symptomatic $S O D 1^{G 93 A}$ mice at the age of 130 days and age-matched SOD ${ }^{W T}$ mice. Confocal images show NICD-1 expression in the DAPI + nuclei of GFAP + astrocytes, both in resting astrocytes of $S O D 1^{W T}$ mice and diseased and reactive astrocytes of symptomatic $S O D 1^{G 93 A}$ mice. Scale bar is $10 \mu \mathrm{m}$. cord of $S O D 1^{G 93 A}$ mice. To test this, we evaluated Notch receptor cleavage, the expression of key-components of the downstream transcription activation complex and the expression of Notch target genes.

NICD-1 was readily detectable by immunohistochemical staining using tyramide signal amplification, although it was undetectable using Western blot. In order to quantify Notch-1 activation in a cell-specific way, we evaluated the number of NICD-1 postive cells in the affected lumbar spinal cord ventral horn of SOD $1{ }^{\mathrm{G} 931}$ mice compared to control ventral horn of $S O D 1^{W T}$ mice. Unfortunately, the lack of specific antibodies precludes the study of NICD-2. As shown in Fig. 2A, nuclear NICD-1 staining was present in a significantly higher number of cells in the lumbar spinal cord ventral horn of symptomatic SOD $1^{G 93 A}$ mice compared to age-matched $S O D 1^{W T}$ control mice (Fig. $2 \mathrm{~A}$ ). The specificity of the NICD-1 staining was evaluated by staining the spinal cord of Notch- $1^{\text {lox/lox }}$ CAGG-CreER mice (Supplementary Fig. 2A-B), in which Notch-1 mRNA expression is reduced by approximately $90 \%$ upon tamoxifen administration (Supplementary Fig. 2C), resulting in an almost complete abolishment of NICD-1 positive cells in the ventral horn (Supplementary Fig. 2A-B).

We next evaluated the expression levels of Rbpjk and Maml. These cofactors form the transcription-activation complex together with NICD and contribute to enhanced Notch signaling activation when expressed at high-level (Larabee et al., 2013; Kochert et al., 2011). A significant upregulation of Rbpjk and all three isoforms of Maml (Maml-1, Maml-2, Maml-3) was evident during disease progression, when compared to age-matched control SOD $1^{W T}$ mice or non-transgenic mice (Fig. 2B). In agreement with the mRNA data, protein expression of RBPjk was increased as well (Fig. 2C-D).

We next quantified the expression of the Hes and Hey downstream target genes of Notch, of which the expression levels are differentially affected upon activation of Notch signaling (Petrovic et al., 2015; Hatakeyama et al., 2004; Mukhopadhyay et al., 2009; Fior \& Henrique, 2005). Indeed, Hes-7 and Hey-2 expression levels were significantly enhanced during disease progression (Fig. 2E), while levels of Hes-1 and Hey-1 remained unchanged (Fig. 2F), and Hes-5 and Hey-L expression levels were decreased (Fig. 2G). This pattern of changes of target gene expression is known to occur upon Notch signaling activation (Petrovic et al., 2015; Hatakeyama et al., 2004; Mukhopadhyay et al., 2009; Fior \& Henrique, 2005).

Overall our data suggest that the Notch signaling pathway is overactive in the lumbar spinal cord of ALS mice compared to controls.

\subsection{Neurons and astrocytes are the cell types involved in Notch signaling in SOD $1^{\text {G93A }}$ mice}

To investigate which cell types of the spinal cord mediate Notch signaling, we determined the cellular localization of NICD-1 and JAGGED-1 using double staining for these proteins and different neuronal and glial cell type markers. In the spinal cord ventral horn of both $S O D 1^{G 93 A}$ mice at the age of 130 days (symptomatic stage) and agematched SOD $1^{W T}$ mice, NICD-1 was found in nuclei of GFAP-positive astrocytes, and more NICD-1 positive astrocytes were observed in the ventral horn of symptomatic SOD $1^{G 93 A}$ mice when compared to control $S O D 1^{W T}$ mice (Fig. 3 and supplementary Fig. 3). No NICD-1 signal was observed in NeuN-positive neurons (Supplementary Fig. 4A and D), and only some of the CC1-positive oligodendrocytes and the F4-80-positive reactive microglia are positive for NICD-1 (Supplementary Fig. 4B-D), however as described before (Zhang et al., 2014), the levels of NICD-1 in microglia and oligodendrocytes are considered negligible. Consequently, our data suggest that the increased Notch signaling activation in the spinal cord of $S O D 1^{G 93 A}$ mice is mainly astrocytic in nature. JAGGED-1 expression was clearly identifiable in NeuN-positive (motor) neurons, both in the $S O D 1^{W T}$ and $S O D 1^{G 93 A}$ mouse spinal cord (Fig. 4A). Interestingly, after disease onset, JAGGED-1 was abundantly expressed in GFAP-positive reactive astrocytes in symptomatic $S O D 1^{G 93 A}$ mice at the age of 130 days (Fig. 4B), while never observed in 
A
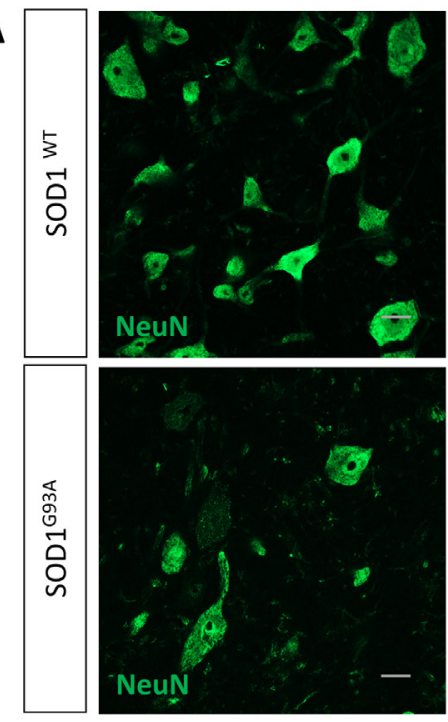

B

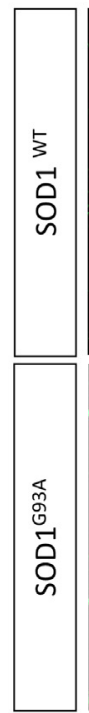

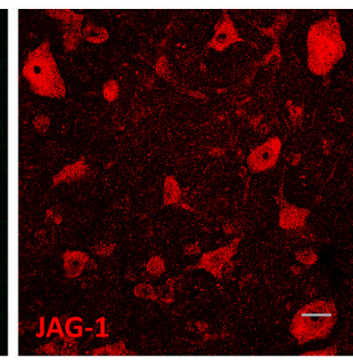
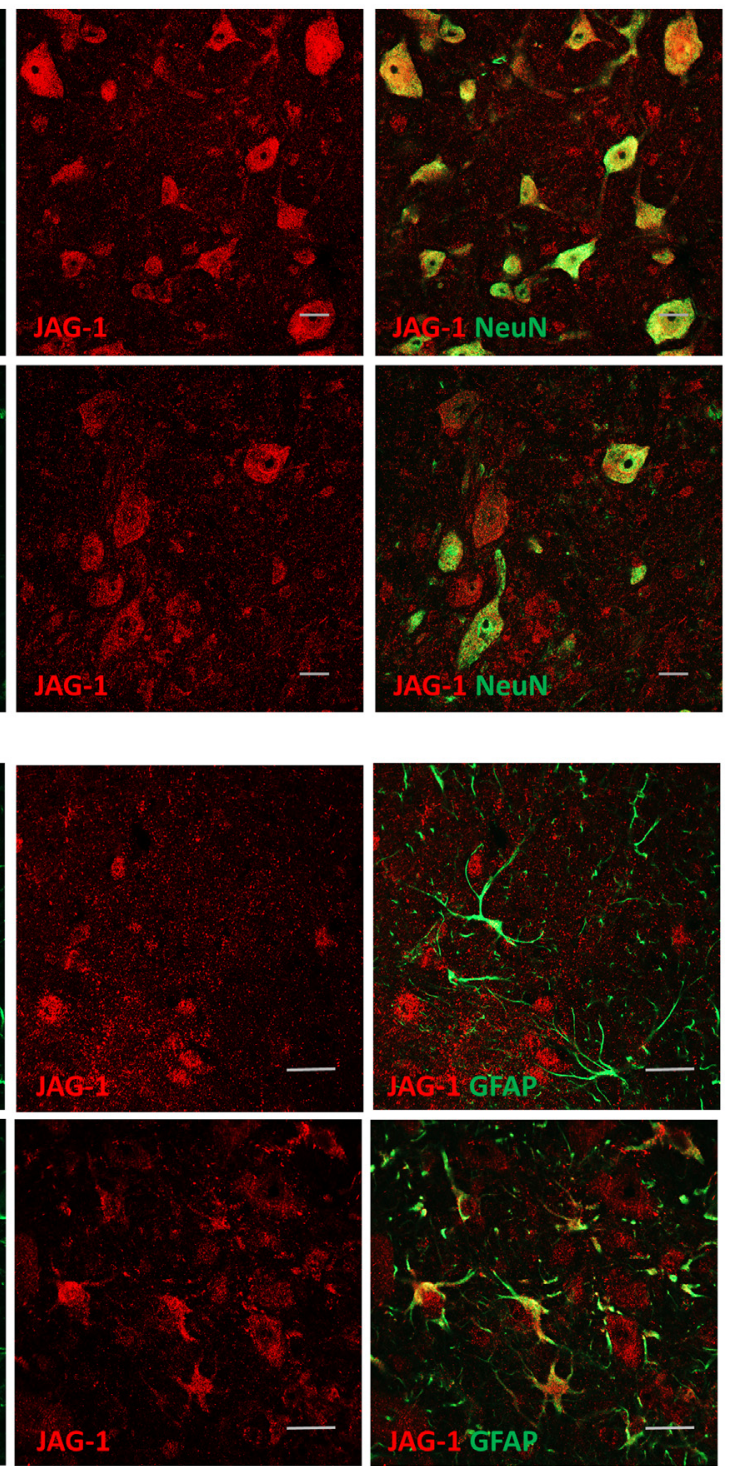

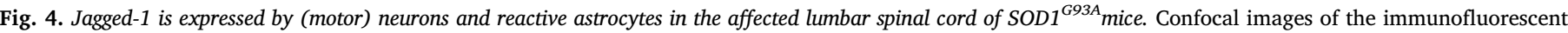

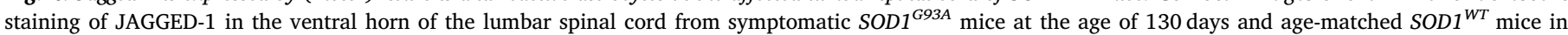
combination with (A) the neuronal marker NeuN and (B) the astrocytic marker GFAP. Scale bar is $20 \mu \mathrm{m}$.

astrocytes of non-transgenic or $S O D 1^{W T}$ mice. The typical staining pattern of JAGGED-1 disappeared after pre-incubation of the JAGGED1 antibody with two different neutralizing JAGGED-1 peptides, sc-6011 P (Santa Cruz Biotechnology) and NBP1-90208 PEP (Novus Biologicals), demonstrating the specificity of the antibody (Supplementary Fig. 5).

These data reveal prominent activation of Notch signaling and abundant expression of JAGGED-1 in spinal cord reactive astrocytes of symptomatic ALS mice.

\subsection{Aberrant Notch signaling in postmortem tissue from ALS patients}

To examine whether enhanced Notch-1 signaling is also present in human ALS, we investigated the Notch signaling pathway in postmortem spinal cords from sALS patients and compared them to spinal cords from control individuals. From publically available data (www.ncbi. nlm.nih.gov/geo, accession number GSE18920) (Rabin et al., 2010) we retrieved the mRNA expression of NOTCH-1, NOTCH-2 and JAGGED-1 in human lumbar spinal cord motor neurons (obtained using laser capture microdissection) and in the remaining anterior horn (devoid of motor neurons) from 12 sALS patients and 8 control individuals.
Increased mRNA expression of NOTCH-1, NOTCH-2 and JAGGED-1 was found in sALS spinal cords, both in the motor neuron fraction (Fig. 5A), and in the remaining anterior horn fraction (Fig. 5B). No significantly altered mRNA expression was observed for the other Notch receptors and ligands, except for the non-canonical ligand F3/CONTACTIN-1 that was more abundant in sALS spinal cords (Supplementary Fig. 6A-B).

We then evaluated the Notch signaling activation status by studying the mRNA expression profiles of the cofactors RBPjk and MAML, and the Notch target genes. In the motor neuron fraction of sALS patients, a significant increase in mRNA expression of the cofactor $M A M L-2$ and the Notch target genes HES-1 and HEY-2 was observed when compared to human control motor neurons (Fig. 5A). In the remaining anterior horn fraction, the mRNA expression of HES-1 and HEY-2 tended to be increased as well, but to a lesser degree than in the motor neuron fraction. Other components of the Notch pathway did not show consistent changes of expression (Supplementary Fig. 6A-B). The purity of the motor neuron fraction and the remaining anterior horn glial fraction and the presence of reactive astrocytosis and microgliosis in the sALS samples compared to the control samples, were analyzed by evaluating the expression of the motor neuronal marker choline acetyltransferase (ChAT) and the glial markers glial fibrillary acidic protein 
A

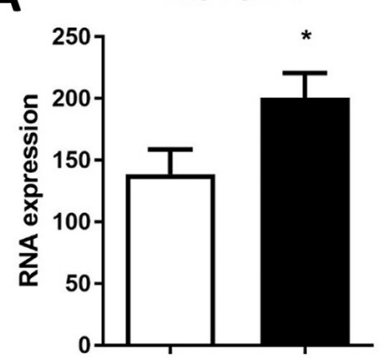

$R B P j$

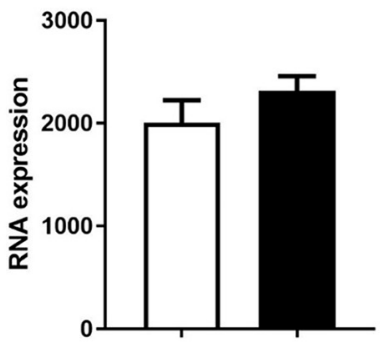

NOTCH-2

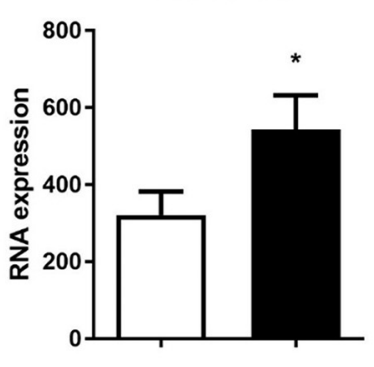

MAML-2

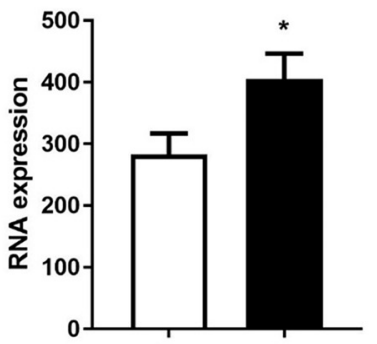

JAGGED-1

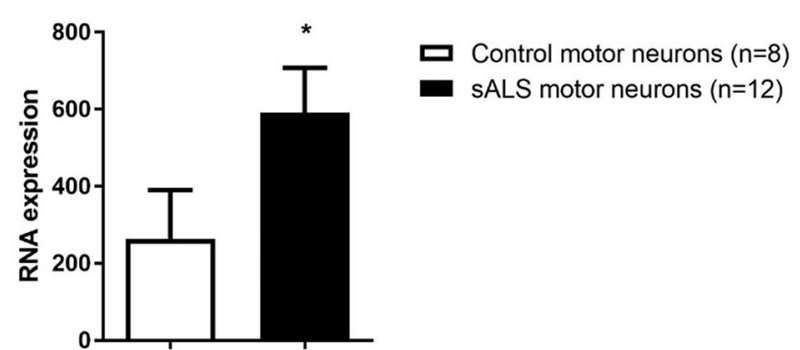

HES-1
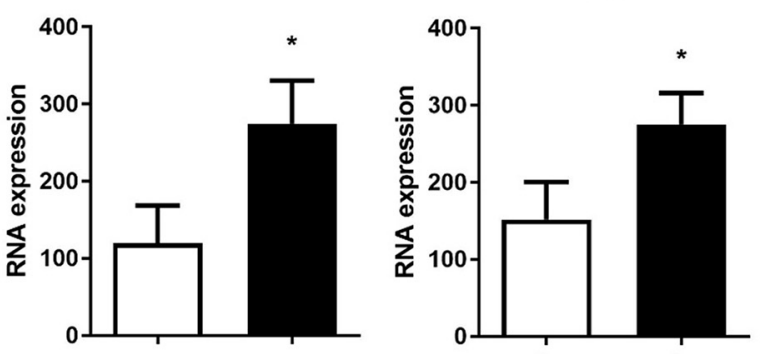

B

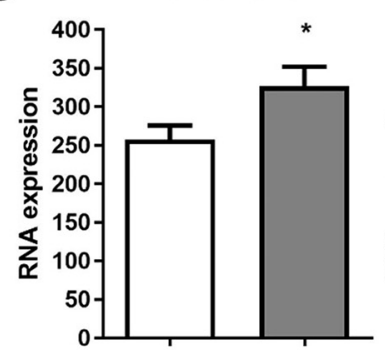

$R B P j$

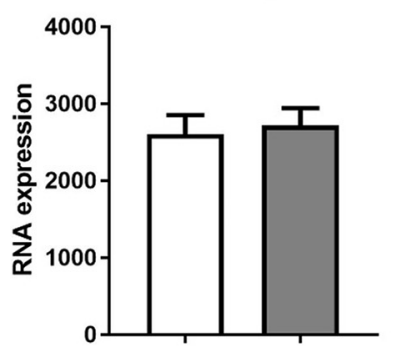

NOTCH-2

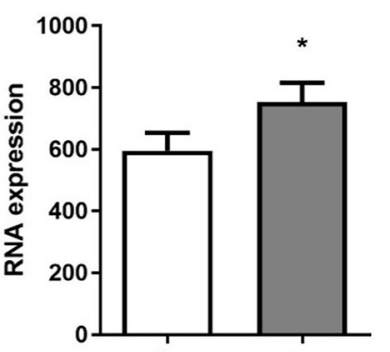

MAML-2

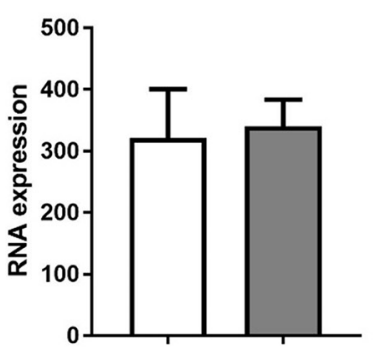

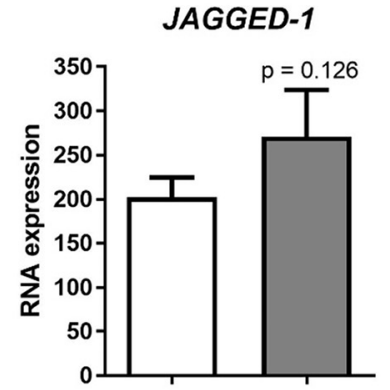

HES-1

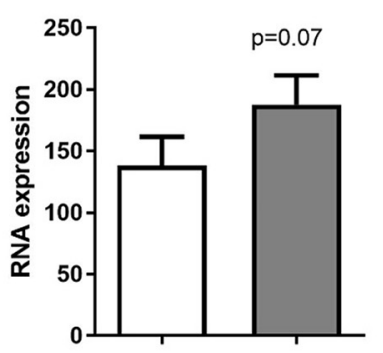

드니이 anterior horn $(n=8)$

口 sALS anterior horn $(n=12)$

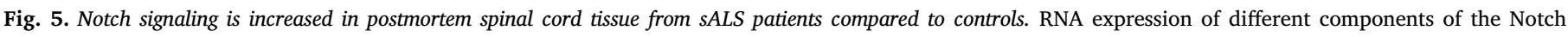

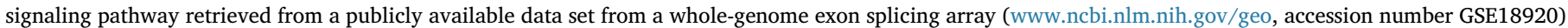

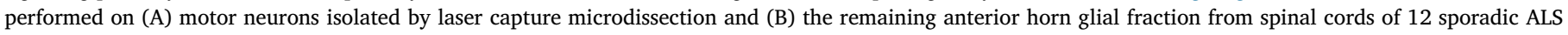

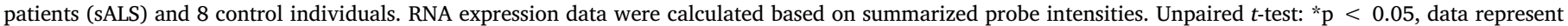
mean \pm SEM.

(GFAP) for astrocytes and ionized calcium-binding adapter molecule-1 (IBA-1) for microglia (Supplementary Fig. 7).

We next examined the cellular localization of JAGGED-1 and NICD1 in postmortem spinal cord of sALS patients and control individuals (Fig. 6). In both ALS and control spinal cord, JAGGED-1 was faintly expressed in (motor) neurons. In agreement with what we observed in the $S O D 1^{G 93 A}$ mouse model we observed JAGGED-1 to be expressed by reactive astrocytes in sALS lumbar spinal cord samples, while JAGGED1 was absent in resting astrocytes in lumbar spinal cord samples from control individuals. NICD-1 could not be detected in the lumbar spinal cord ventral horn of control individuals. However, in the lumbar spinal cord ventral horn of sALS patients NICD-1 was prominently present in the reactive astrocytes. We observed this astrocytic JAGGED-1 and NICD-1 staining pattern in 5 out of 6 sALS patient lumbar spinal cords. No staining signal was observed in the sALS patient sample with the longest postmortem time interval (PMI $=8.5 \mathrm{~h})$.

Together, these data obtained from sALS patients are in full agreement with our findings from the SOD $1^{G 93 A}$ mouse model, and confirm the increased Notch signaling activation and the aberrant cellular localization of JAGGED-1 and NICD-1 in reactive astrocytes in sALS spinal cord. 

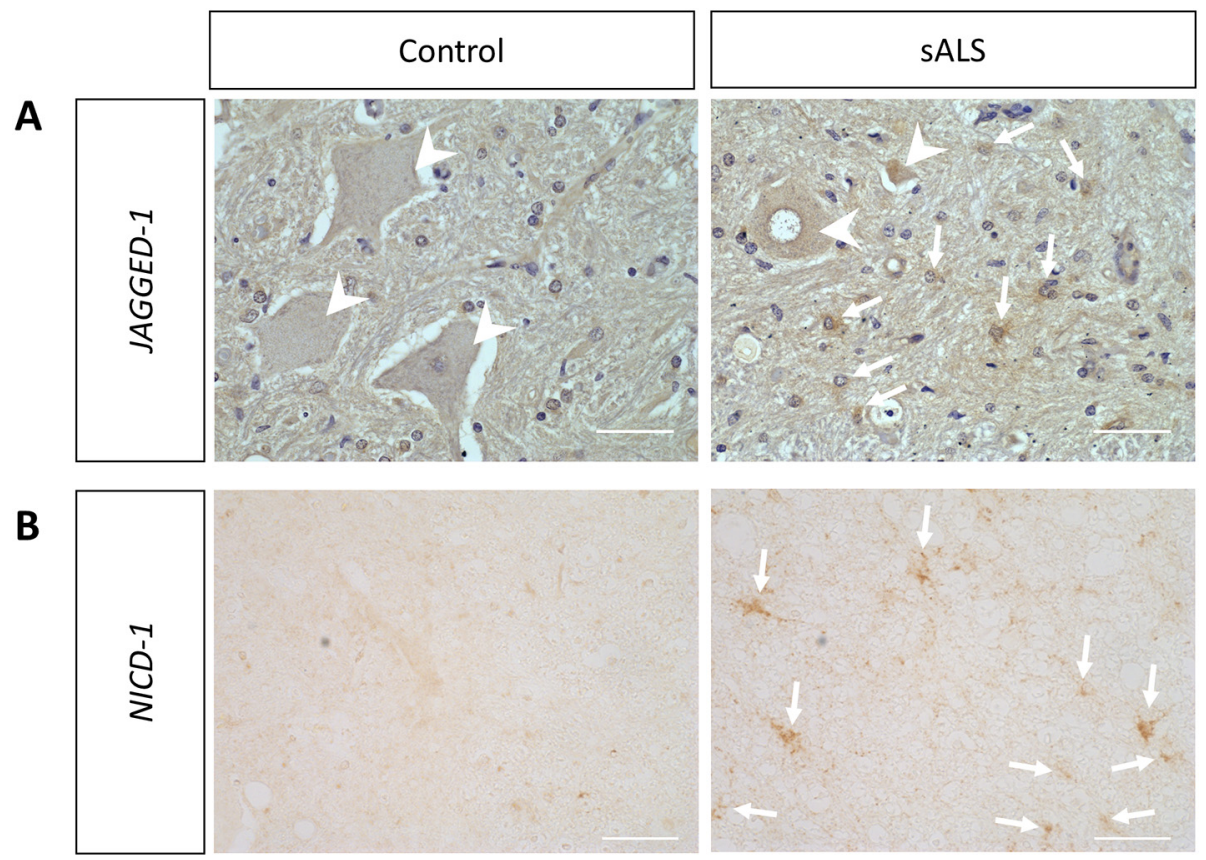

C
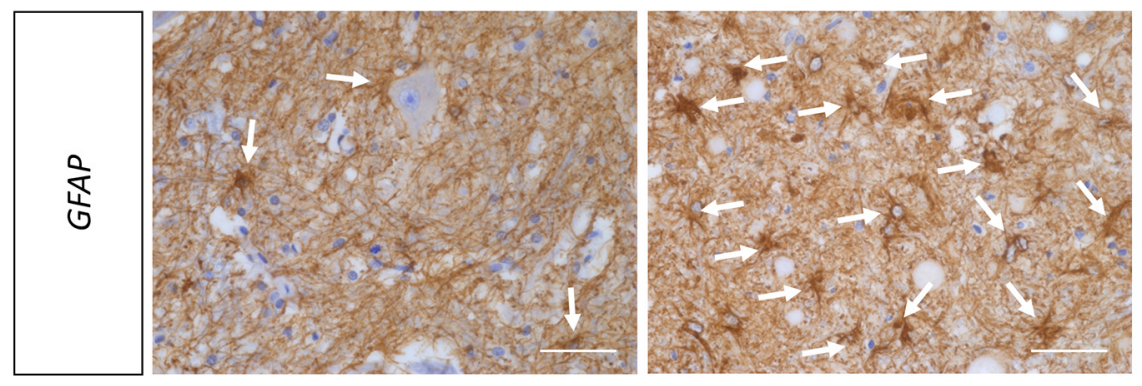

Fig. 6. JAGGED-1 is expressed in (motor) neurons and reactive astrocytes in postmortem spinal cord from ALS patients, and NICD-1 is expressed in reactive astrocytes only. Representative images of the immunohistochemical staining of (A) JAGGED-1, (B) NICD-1 and (C) GFAP in the ventral horn of postmortem human spinal cords from control individuals (left) and sALS patients (right). Arrowheads indicate the faint JAGGED-1 expression in (motor) neurons and the arrows indicate the pronounced JAGGED-1, NICD-1 and GFAP expression in the reactive astrocytes. Scale bar is $50 \mu \mathrm{m}$ and $\mathrm{n}=6$ individuals per group.

\subsection{Deleting Jagged-1 in astrocytes aggravates disease progression of SOD $1^{G 93 A}$ mice}

We next investigated whether interfering with the Notch signaling system may affect motor neuron degeneration in the ALS mouse model. As Jagged-1 is the only Notch ligand showing alterations in expression level in spinal cord of sALS patients and SOD $1^{G 93 A}$ mice, and is prominently expressed in reactive ALS-affected astrocytes, but not in resting healthy astrocytes, we aimed to study the role of the ligand Jagged-1 in reactive astrocytes in ALS. Therefore, we inactivated Jagged-1 specifically in astrocytes by crossbreeding Jagged-1 ${ }^{\text {lox/lox }}$ mice with Cx30-CreER mice. In these transgenic mice, exon 4 of the ligand Jagged-1 is deleted by Cre-mediated excision upon administration of tamoxifen (Tx) at the age of 60 days (asymptomatic). This modified allele gives rise to a truncated non-functional JAGGED-1 protein lacking the Notch receptor binding domain (Kiernan et al., 2006).

The specificity and efficiency of the astrocytic Cx30-CreER line was evaluated by crossbreeding Cx30-CreER mice to YFP reporter mice (Rosa- ${ }^{l o x} S T O P^{l o x}$-YFP mice), showing the expression of YFP upon tamoxifen induced Cre-mediated excision of the STOP-cassette. Immunofluorescent co-staining of YFP and GFAP in the spinal cord of Rosa $^{\text {lox }}{ }^{-}$TOP $^{\text {lox }}$-YFP Cx30-CreER mice after tamoxifen administration $(Y F P+/+C x 30-C r e E R \&$ Tx $)$ revealed that YFP was only expressed in GFAP-positive astrocytes and that recombination took place in on average $84 \pm 5 \%$ of all GFAP-positive astrocytes (Supplementary fig. 8A-B). No recombination took place in the absence of tamoxifen (YFP
$+/+$ Cx30-CreER \& no Tx) or in the absence of Cx30-CreER (YFP + / + $\& \mathrm{Tx}$ ) (Supplementary Fig. 8A-B).

The offspring of the breeding of Jagged-1 ${ }^{\text {lox } / \text { lox }}$ mice with Cx30CreER mice and $S O D 1^{G 93 A}$ mice was treated with tamoxifen at the age of 60 days. This tamoxifen-induced deletion of exon 4 of Jagged-1 in astrocytes resulted in a significant reduction of total spinal cord mRNA of Jagged-1 containing exon 4 of on average $17.5 \pm 3 \%$ in Jagged-1 ${ }^{\text {lox } /}$

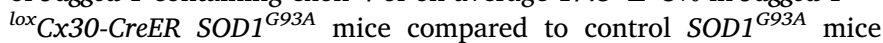
$\left(S O D 1^{G 93 A}+\mathrm{Tx}\right)$ (Supplementary fig. $8 \mathrm{C}$ ). The remaining expression of Jagged-1 containing exon 4 thus largely came from non-astrocytic (mainly neuronal) cells. No gross phenotypical abnormalities were observed in these mice upon inactivation of Jagged-1 in double trans-

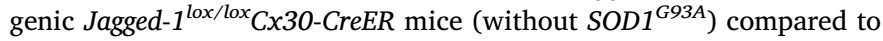
control Jagged-1 ${ }^{\text {lox/lox }}$ mice (data not shown).

Astrocytic homozygous inactivation of Jagged-1 in $S O D 1^{G 93 A}$ mice (Jagged- $1^{\text {lox } / l o x} C x 30$-CreER SOD $1^{G 93 A}+\mathrm{Tx}$ ), had no effect on disease onset compared to control SOD1 $1^{G 93 A}$ mice (SOD1 $\left.1^{G 93 A}+\mathrm{Tx}\right)($ Fig. $7 \mathrm{~A})$, but accelerated disease after onset. Survival was significantly reduced by 18 days (Fig. 7B) and consequently disease progression was hastened with an on average disease duration of $38 \pm 5$ days in $S O D 1^{G 93 A}$ mice with homozygous Jagged-1 inactivation (Jagged-1 ${ }^{\text {lox } / \text { lox } C \times 30-C r e E R}$ $\left.S O D 1^{G 93 A}+\mathrm{Tx}\right)$ compared to $52 \pm 3$ days in control $S O D 1^{G 93 A}$ mice (Fig. 7C). Follow-up of body weight during disease progression (Fig. 7D) showed significantly more pronounced weight loss when inactivating Jagged-1 homozygously in astrocytes compared to control $S O D 1^{G 93 A}$ mice. Motor performance was assessed by two classical tests, 
A

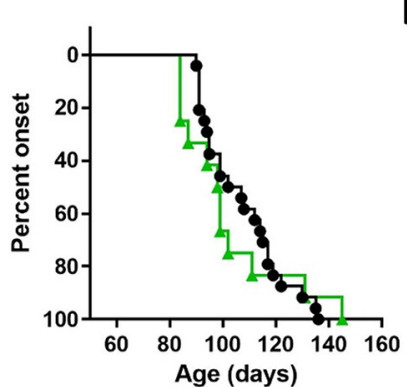

D

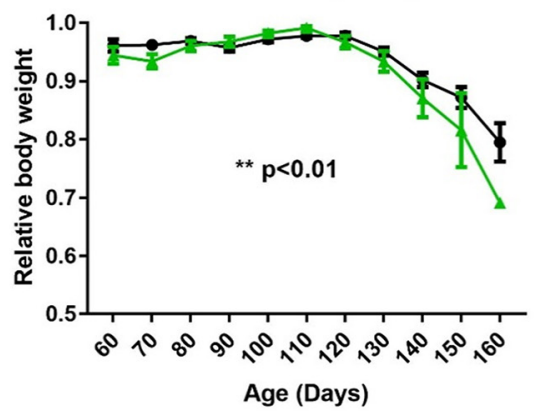

$\mathbf{F}$

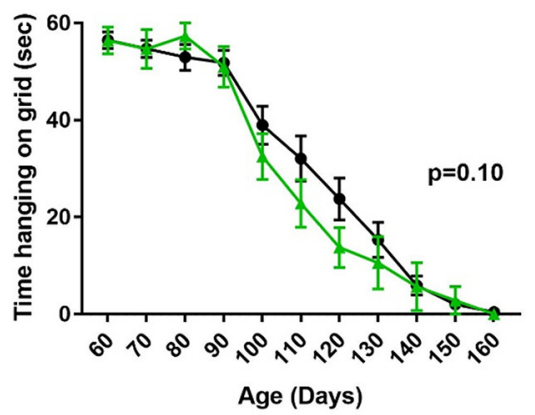

H

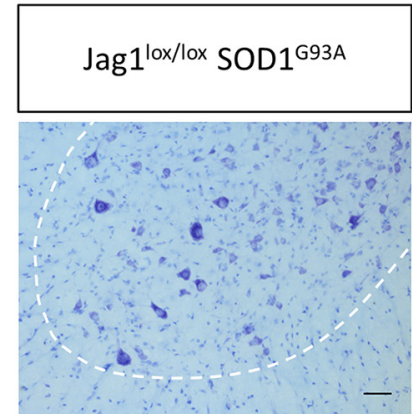

B

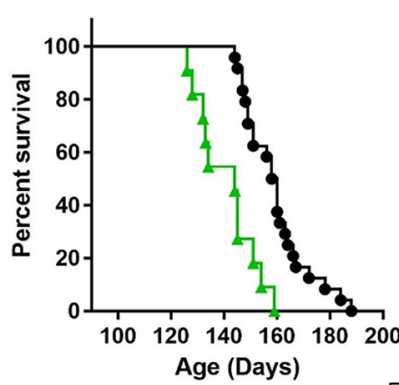

E

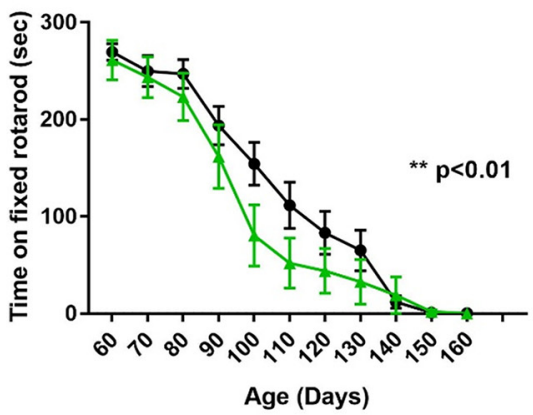

G

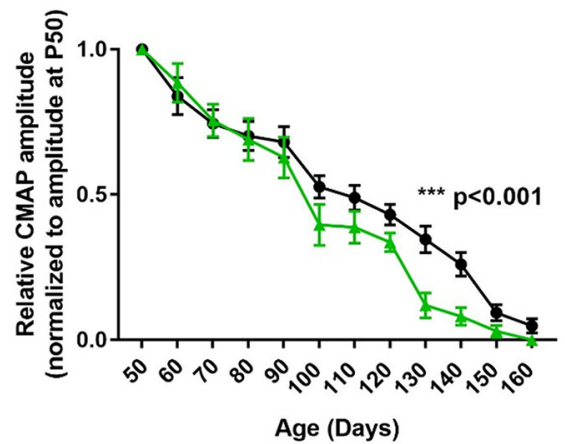

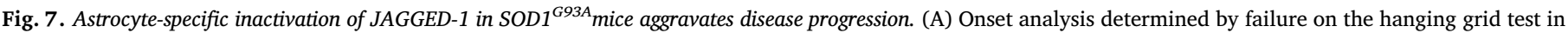

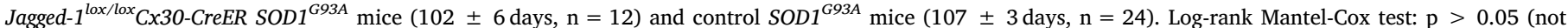

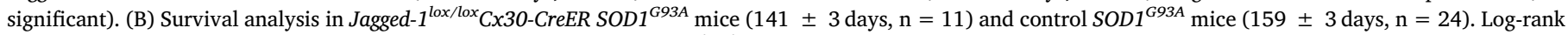

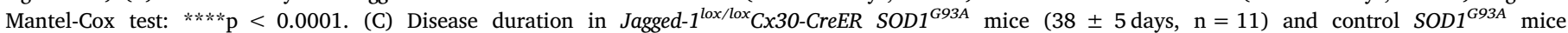

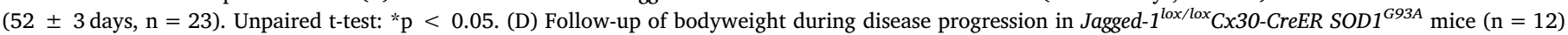

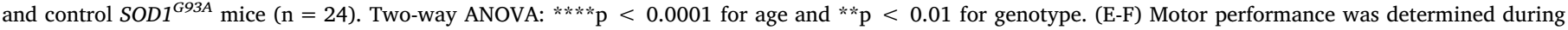

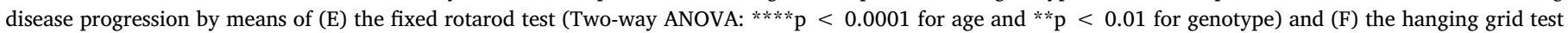

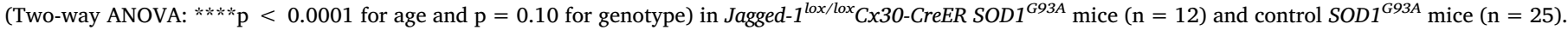

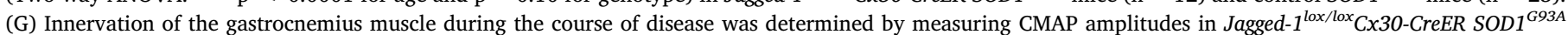

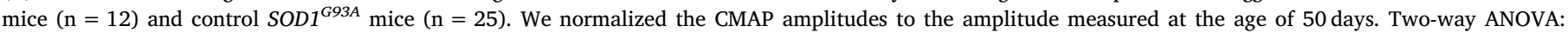

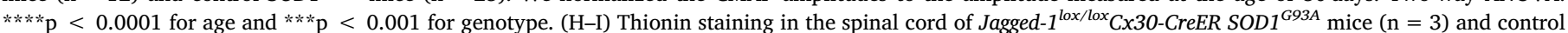

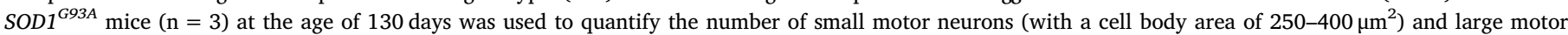

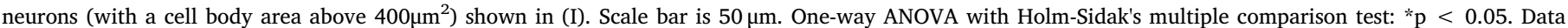
represent mean \pm SEM. All mice received tamoxifen at the age of 60 days. 
the fixed rotarod test (Fig. 7E) and the hanging grid test (Fig. 7F). We observed that $S O D 1^{G 93 A}$ mice with homozygous astrocyte-specific Jagged-1 inactivation perform significantly worse on the fixed rotarod tests compared to control SOD $1^{G 93 A}$ mice (Fig. 7E). With the hanging grid test we could detect a clear tendency towards reduced performance (Fig. 7F). Innervation of the gastrocnemius muscle was evaluated by measuring compound muscle action potentials (CMAP) at the level of the gastrocnemius muscle (Fig. 7G). We observed that the CMAP amplitudes decreased significantly faster during disease progression, when Jagged-1 was inactivated homozygously in astrocytes of $S O D 1^{G 93 A}$ mice, compared to control $S O D 1^{G 93 A}$ mice (Fig. $7 \mathrm{G}$ ). No difference in CMAP latencies was noticed (data not shown).

Quantification of the number of motor neurons revealed that both the number of small motor neurons (cell body area of $250-400 \mu \mathrm{m}^{2}$ ) and the number of large motor neurons (cell body area above $400 \mu \mathrm{m}^{2}$ ) were significantly reduced in the lumbar spinal cord ventral horn of Jagged- $1^{\text {lox/lox } C x 30-C r e E R ~ S O D 1}{ }^{\text {G93A }}$ mice at the age of 130 days, when compared to age-matched littermate control SOD $1^{G 93 A}$ mice (Fig. $7 \mathrm{H}-\mathrm{I}$ ). We additionally investigated whether this enhanced disease severity was evident when quantifying reactive astrogliosis and microgliosis by staining for the markers glial fibrillary acidic protein (GFAP) and ionized calcium-binding adapter molecule 1 (IBA-1) respectively, and although not statisticaly significant, we observed a $38 \%$ increase in GFAP staining intensity and a $53 \%$ increase in IBA- 1 staining in the lumbar spinal cord ventral horn of Jagged- $1^{\text {lox } / \text { lox } C x 30-C r e E R ~ S O D ~} 1^{\text {G93A }}$ mice at the age of 130 days compared to control SOD $1^{G 93 A}$ mice (Supplementary Fig. 9).

Heterozygous astrocytic inactivation of Jagged-1 in SOD $1^{G 93 A}$ mice (Jagged-1 ${ }^{\text {lox/- }}$ Cx30-CreER SOD $1^{G 93 A}+\mathrm{Tx}$ ) resulted in a more modest reduction of total spinal cord mRNA of Jagged-1 containing exon 4 (on average $9.2 \pm 2 \%$ ) as expected (data not shown) and did not affect disease onset or motor performance. Of interest, this limited reduction already tended to reduce survival (reduction of 12 days) compared to control SOD $1^{G 93 A}$ mice (SOD $1^{G 93 A}+\mathrm{Tx}$ ) (data not shown).

We next evaluated the effect of astrocytic Jagged-1 inactivation on Notch signaling in this cell type. To this end, we stained for NICD-1 and quantified the number of NICD-1 positive nuclei in the lumbar spinal cord ventral horn of end stage SOD $1^{G 93 A}$ mice and end stage SOD $1^{G 93 A}$ mice with homozygous inactivation of Jagged-1 specificly in astrocytes (Fig. 8A-B and supplementary Fig. 10). We found the number of such NICD-1 positive nuclei to be significantly increased with $80 \%$ in Jagged-

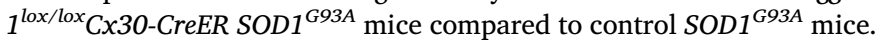

Together these data show that homozygous inactivation of Jagged-1 specifically in astrocytes of $S O D 1^{G 93 A}$ mice aggravates disease after onset, shortens survival and enhances Notch signaling activation, thereby suggesting a beneficial role for the astrocytic expressed Jagged1.

\section{Discussion}

The Notch signaling pathway, as a critical mediators of cell-cell interaction (Artavanis-Tsakonas et al., 1999; Lai, 2004), is an underestimated target in the study of the non-cell autonomous component of the pathogenesis of ALS and other neurodegenerative diseases. This is mainly due to the impressive complexity of this pathway and the rather limited knowledge about its role in the adult central nervous system (Ables et al., 2011).

In this study, we explored the contribution of Notch signaling to the pathogenesis of ALS, by using sALS patient samples, the SOD1 ${ }^{\text {G93A }}$ mouse model and conditional knockout mice. Our data show that the Notch signaling pathway is abnormally active in the affected spinal cord of both sALS patients and SOD ${ }^{G 93 A}$ mice and contributes to motor neuron degeneration and disease progression in the ALS mouse model. Several lines of evidence support this statement.

First, overactive Notch signaling was suggested by the prominent increase in the mRNA expression of the receptors Notch-1 and Notch-2, along with an increase in the expression of the processed (not fulllength) forms of these Notch proteins, including an increase in the levels of the active signaling fragment NICD-1, in $S O D 1^{G 93 A}$ mice. In human sALS postmortem lumbar spinal cord, the mRNA expression level of these two Notch receptors was also found to be increased in both the motor neurons and the remaining non-motor neuron fraction, as well as overactive Notch signaling was suggested by the increase in the levels of the active signaling fragment NICD-1 in sALS postmortem lumbar spinal cord ventral horn, when compared to control individuals.

Second, the increased mRNA expression of the canonical ligand Jagged-1 in lumbar spinal cord of both sALS patients and SOD1 ${ }^{G 93 A}$ mice in combination with the decreased expression level of the full length form of the Jagged-1 protein in the spinal cord of $S O D 1^{G 93 A}$ mice, suggests increased JAGGED-1 processing and turnover (cleavage, endocytosis and degradation) after signal transduction (D'Souza et al., 2008; Hansson et al., 2010; He et al., 2014; Lavoie \& Selkoe, 2003; Le Bras et al., 2011).

Furthermore in lumbar spinal cord of $S O D 1^{G 93 A}$ mice and sALS patients, several cofactors needed to form the transcription activation complex togheter with the active signaling fragment NICD, are found to be upregulated. This observation suggests increased Notch signaling activation as well, because it is known from overexpression studies that increased levels of RBPjk or Mastermind-like 2 are sufficient to trigger Notch signaling activation (Larabee et al., 2013; Kochert et al., 2011).

We visualized and quantified NICD-1, along with the expression levels of downstream Notch target genes to evaluate the activation status of the Notch signaling pathway. Significantly more astrocytes stained positive for NICD-1 in the lumbar spinal cord ventral horn of both SOD $1^{G 93 A}$ mice and sALS patients compared to controls, reflecting increased signaling activation. Surprisingly, we could not detect NICD-1 in neurons and only very few oligodendrocytes stained positive for NICD-1, in spite of the reported effects of Notch-1 in neurons (Bonini et al., 2013; Berezovska et al., 1999; Redmond et al., 2000; El Bejjani \& Hammarlund, 2012) and oligodendroglial cells (Wang et al., 1998; Genoud et al., 2002; Popko, 2003; Park \& Appel, 2003). Obviously, its level may be below the limit of detection by immunohistochemistry. These results are in agreement with publically available data (https:// web.stanford.edu/group/barres_lab/brain_rnaseq.html) (Zhang et al., 2014). When looking at the Notch target genes in SOD1 ${ }^{G 93 A}$ mice compared to $S O D 1^{W T}$ mice, a differential effect on target gene expression was observed, with enhanced expression of Hes-7 and Hey-2, decreased expression of Hes-5 and Hey-L, and unchanged Hes- 1 and Hey-1 levels. This is known to occur upon Notch signaling activation, as they are subject to a rather complex system of regulation (Petrovic et al., 2015; Hatakeyama et al., 2004; Mukhopadhyay et al., 2009; Fior \& Henrique, 2005). The Hes and Hey genes described above are all direct Notch downstream targets, but they differ in the level of Notch activation required for their transcription activation and their expression is known to correlate with different Notch ligands (Petrovic et al., 2015). In addition they also differ in mRNA stability (Petrovic et al., 2015), they can cross-regulate each other (Petrovic et al., 2015; Hatakeyama et al., 2004; Mukhopadhyay et al., 2009; Fior \& Henrique, 2005) and their expression can be influenced by other signaling pathways, such as Bmp, Wnt and Fgf signaling (Petrovic et al., 2015). In contrast, the increase in Notch signaling activation in the spinal cord of sALS patients is nicely reflected in the expression of the Notch target genes, with an upregulation of HES-1 and HEY-2, while no changes were observed for HES-7, HEY-1 and HEY-L. HES-5 was not detected in the human dataset. The differential expression of the target genes between the spinal cord of the ALS mouse model and sALS patients can be explained by interspecies-variability and possibly also by the fact that we found the noncanonical ligand F3/CONTACTIN-1 to be significantly upregulated in the motor neurons and the non-motor neuron fraction of sALS patient spinal cord samples, while we could not detected a significant increase for $F 3 /$ Contactin-1 in the spinal cord of $S O D 1^{G 93 A}$ mice.

Overal these data obtained from the ALS mouse model and 
A

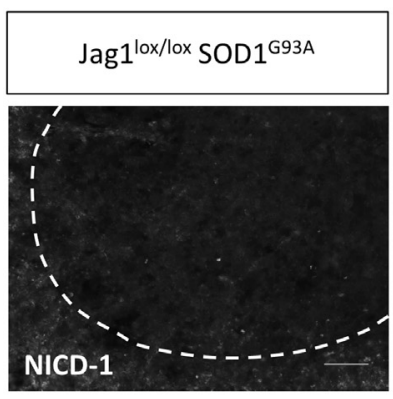

B

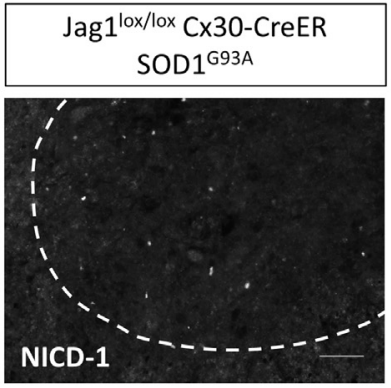

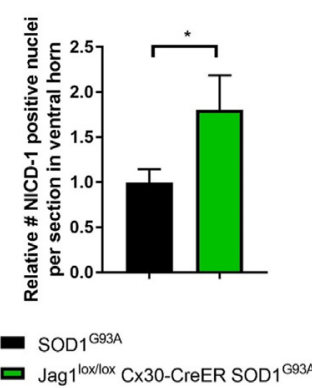

C
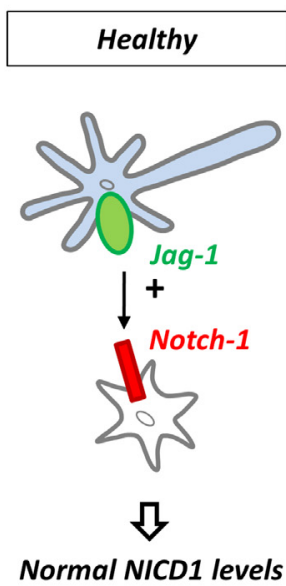
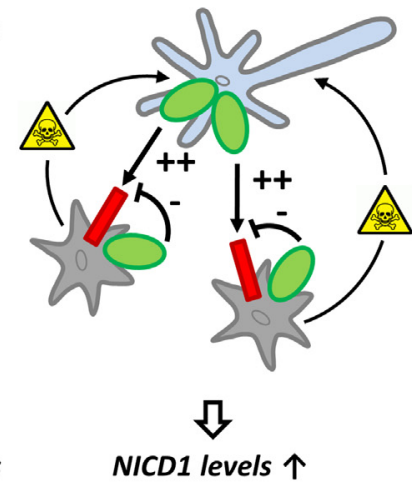

Astrocytic Jag-1 inactivation in ALS

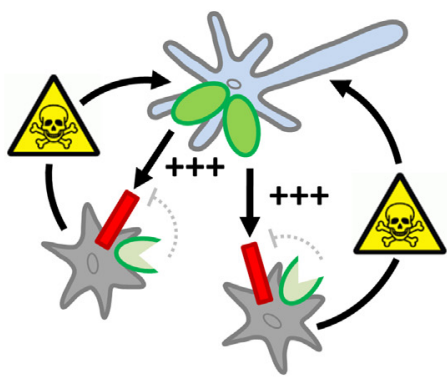

$\sqrt{3}$

Fig. 8. Astrocyte-specific inactivation of JAGGED-1 in SOD $1{ }^{G 93 A}$ mice enhances Notch signaling activation in lumbar spinal cord ventral horn, possibly due to the removal of Jagged-1 mediated cis-inhibition. (A) Representative image of the immunofluorescent staining of NICD-1 in the ventral horn of lumbar spinal cord of end stage Jagged-1 ${ }^{\text {lox/lox }}$ Cx30-CreER $S O D 1^{G 93 A}$ mice $(\mathrm{n}=4)$ and end stage control $S O D 1^{G 93 A}$ mice $(\mathrm{n}=4)$. (B) Quantification of the number of NICD-1 positive nuclei per section in the lumbar spinal cord ventral horn. Nuclei were stained with DAPI (see supplementary fig. 10 for images and overlay). Scale bar is $50 \mu \mathrm{m}$. Un-paired t-test: ${ }^{*} \mathrm{p}<0.05$. Data represent mean \pm SEM. All mice received tamoxifen at the age of 60 days. (C) Model describing our hypothesis about the role of Jagged-1 expressed on reactive astrocytes in ALS. In healthy spinal cord (left panel), Jagged-1 is expressed on (motor) neurons and Notch-1 on some astrocytes, resulting into basal levels of NICD-1, reflecting normal Notch signaling activation (indicated by “+”). In ALS affected spinal cord (middle panel), Jagged-1 is expressed on (motor) neurons and more astrocytes express Notch-1, resulting into an increase in the number of NICD-1 positive astrocytes, reflecting enhanced Notch signaling activation (indicated by " ++ ") that is harmful for motor neurons in ALS. Remarkably, reactive ALS astrocytes overexpress Jagged-1 as well. Ligands expressed on the same cell as the Notch receptor act inhibitory on Notch signaling activation, a mechanism called cisinhibition. Consequently, we believe that the astrocytic Jagged-1 mediates cis-inhibition (indicated by “-”) as an attempt to reduce the enhanced Notch signaling activation in ALS, but it is not enough to

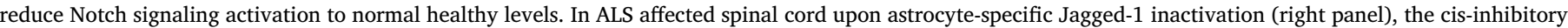

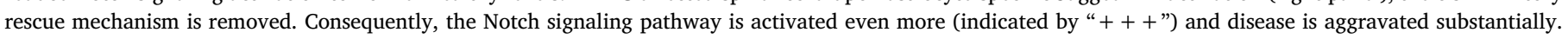

postmortem sALS patient samples clearly demonstrate a signature of overactive Notch signaling in the lumbar spinal cord.

Interestingly, we found Jagged-1 to be ectopically expressed in the reactive ALS-astrocytes localized in the lumbar spinal cord ventral horn of both sALS patients and $S O D 1^{G 93 A}$ mice, while it was below detection level in resting, healthy astrocytes of control individuals and SOD $1^{W T}$ mice. This observation together with our finding of Jagged-1 to be the only canonical ligand showing increased mRNA expression levels in both $S O D 1^{G 93 A}$ mice and sALS patients suggests its involvement in the pathogenesis of ALS and inspired us to study the role of Jagged-1 on these reactive ALS-astrocytes. Inactivation of Jagged-1 specifically in astrocytes aggravated disease substantially by affecting disease duration rather than disease onset, which is in agreement with the hypothesis that reactive astrocytes affect disease progression rather than disease onset (Yamanaka et al., 2008; Yamanaka \& Komine, 2018). Interestingly, the worsening of disease upon astrocyte-specific inactivation of Jagged-1, both behaviorally and pathologically, was accompanied by enhanced Notch activation. This can be explained by our model depicted in Fig. 8C, that is based on the fact that the Notch signaling pathway only can get activated by a ligand positioned in trans (i.e. on another cell) (Chillakuri et al., 2012), while it is inhibited by a ligand positioned in cis (i.e. on the same cell) (del Alamo et al., 2011). We suggest that the overactive Notch signaling in astrocytes, possibly activated by neuronal trans-Jagged-1, is detrimental to the motor neuronal system and contributes to the pathogenesis of ALS; reactive astrocytes attempt to mitigate this detrimental trans-activation of the Notch signaling pathway by overexpressing Jagged-1 in order to mediate cis-inhibition (see model in Fig. 8C).

All togheter, this study shows that aberrant Notch signaling activation contributes to motor neuron degeneration in ALS and that exacerbation of Notch signaling by inactivation of the astrocytic Jagged-1 accelerates disease progression after onset. A similar detrimental effect of Notch on neurodegenerative diseases has been suggested before, in a mouse model of focal ischemic stroke showing a significant increase in Notch signaling activation (Arumugam et al., 2006). However, when the NICD levels were decreased, as in mice transgenic for antisense Notch and mice treated with $\gamma$-secretase inhibitors, smaller infarct sizes, reduced microglial activation and lymphocyte infiltraton, and a better functional outcome were observed (Arumugam et al., 2006). This study suggests the involvement of Notch signaling in neuroinflammatory responses in other neurodegenerative diseases as well. Our data are also in agreement with findings in Drosophila, showing that loss-of-function mutations of Notch or of downstream Notch target genes extended the lifespan of TDP-43 transgenic flies (Zhan et al., 2013). It should be noted however that they contrast with another study in Drosophila, in which increased Notch expression was found to suppress toxicity induced by the GR dipeptide repeat associated with C9orf72 expansions (Yang et al., 2015).

Our results also add to the understanding of the role of astrocytes in the pathogenesis of ALS. It is generally accepted that astrocytes actively contribute to the progression of disease (Yamanaka et al., 2008; Yamanaka \& Komine, 2018), because they lose important neuroprotective functions and acquire toxic phenotypes (Yamanaka \& Komine, 2018). However, our results show that reactive ALS astrocytes also exert some protective functions, as they appear to sense the harmfull Notch activation and respond by increasing their own Jagged-1 expression in order to reduce the detrimental Notch signaling activation through Jagged-1 mediated cis-inhibition. Interfering with this protective function of ALS astrocytes therefore deteriorates disease.

It is clear from our data that therapeutic approaches for neurodegenerative disorders that target Notch signaling, although certainly being promissing, have to be looked at with caution, as the balance 
between trans-activation and cis-inhibition needs to be taken into account. Thus, targeting Notch signaling as a therapeutic option has to be done with a great deal of precision in order to circumvent the harmful effects of interfering with feedback mechanisms (Andersson \& Lendahl, 2014).

\section{Acknowledgements}

This work was supported by the Fund for Scientific Research Flanders (FWO; G.0703.12N), the Interuniversity Attraction Poles Programme (P7/16) initiated by the Belgian Science Policy Office, ALS Therapy Alliance, the Association Belge contre les Maladies neuroMusculaires (ABMM) and Research Fund KU Leuven (GOA/11/014). W.R. is supported through the E von Behring Chair for Neuromuscular and Neurodegenerative Disorders and the European Research Council under the European's Seventh Framework Programme (FP7/20072013)/ERC grant agreement ( $n^{\circ}$ 340429). W.R. and P.V.D are supported by the Laevers Fund for ALS Research and the fund 'Een Hart voor ALS'. W.R., P.V.D. and L.V.D.B. are supported by the ALS Liga België and the fund 'Opening the Future'. P.V.D. holds a senior clinical investigatorship from FWO-Vlaanderen. A.Z. was supported by the Fund for Scientific Research Flanders (FWO; G.0786.14 N). A.N. was granted with a $\mathrm{PhD}$ fellowship provided by the Agency for Innovation by Science and Technology in Flanders (IWT). N.C. was granted with a $\mathrm{PhD}$ fellowship provided by FWO-Vlaanderen. S.A.L. is supported by the Petra och Karl Erik Hedborgs Stiftelse, the Swedish FTD initiative, Olle Engkvist Byggmästare Foundation and Åhléns Foundation (mA2/ h17). D.R.T. receives grants from Fund for Scientific Research Flanders (FWO- G0F8516N Odysseus), KU-Leuven (C14/17/107) and Vlaamse Impulsfinanciering voor Netwerken voor Dementie-onderzoek (IWT 135043).

\section{Appendix A. Supplementary data}

Supplementary data to this article can be found online at https:// doi.org/10.1016/j.nbd.2018.07.012.

\section{References}

Ables, J.L., Breunig, J.J., Eisch, A.J., Rakic, P., 2011. Not(ch) just development: notch signalling in the adult brain. Nat. Rev. Neurosci. 12 (5), 269-283.

Andersson, E.R., Lendahl, U., 2014. Therapeutic modulation of notch signalling-are we there yet? Nat. Rev. Drug Discov. 13 (5), 357-378.

Aparicio, E., Mathieu, P., Pereira Luppi, M., Almeira Gubiani, M.F., Adamo, A.M., 2013. The notch signaling pathway: its role in focal CNS demyelination and apotransferrininduced remyelination. J. Neurochem. 127 (6), 819-836.

Appel, S.H., Zhao, W., Beers, D.R., Henkel, J.S., 2011. The microglial-motoneuron dialogue in ALS. Acta Myol. 30 (1), 4-8.

Artavanis-Tsakonas, S., Rand, M.D., Lake, R.J., 1999. Notch signaling: cell fate control and signal integration in development. Science 284 (5415), 770-776.

Arumugam, T.V., Chan, S.L., Jo, D.G., Yilmaz, G., Tang, S.C., Cheng, A., et al., 2006. Gamma secretase-mediated notch signaling worsens brain damage and functional outcome in ischemic stroke. Nat. Med. 12 (6), 621-623.

Arumugam, T.V., Cheng, Y.L., Choi, Y., Choi, Y.H., Yang, S., Yun, Y.K., et al., 2011. Evidence that gamma-secretase-mediated notch signaling induces neuronal cell death via the nuclear factor-kappaB-Bcl-2-interacting mediator of cell death pathway in ischemic stroke. Mol. Pharmacol. 80 (1), 23-31.

Barnabe-Heider, F., Goritz, C., Sabelstrom, H., Takebayashi, H., Pfrieger, F.W., Meletis, K., et al., 2010. Origin of new glial cells in intact and injured adult spinal cord. Cell Stem Cell 7 (4), 470-482.

Beel, S., Moisse, M., Damme, M., De Muynck, L., Robberecht, W., Van Den Bosch, L., et al., 2017. Progranulin functions as a cathepsin D chaperone to stimulate axonal outgrowth in vivo. Hum. Mol. Genet. 26 (15), 2850-2863.

Berezovska, O., Xia, M.Q., Hyman, B.T., 1998. Notch is expressed in adult brain, is coexpressed with presenilin-1, and is altered in Alzheimer disease. J. Neuropathol. Exp. Neurol. 57 (8), 738-745.

Berezovska, O., McLean, P., Knowles, R., Frosh, M., Lu, F.M., Lux, S.E., et al., 1999. Notch1 inhibits neurite outgrowth in postmitotic primary neurons. Neuroscience 93 (2), 433-439.

Boillee, S., Vande Velde, C., Cleveland, D.W., 2006. ALS: a disease of motor neurons and their nonneuronal neighbors. Neuron 52 (1), 39-59.

Bonini, S.A., Ferrari-Toninelli, G., Montinaro, M., Memo, M., 2013. Notch signalling in adult neurons: a potential target for microtubule stabilization. Ther. Adv. Neurol. Disord. 6 (6), 375-385.
Bray, S.J., 2006. Notch signalling: a simple pathway becomes complex. Nat. Rev. Mol. Cell Biol. 7 (9), 678-689.

Brown, R.H., Al-Chalabi, A., 2017. Amyotrophic lateral sclerosis. N. Engl. J. Med. 377 (2), $162-172$.

Caraballo-Miralles, V., Cardona-Rossinyol, A., Garcera, A., Torres-Benito, L., Soler, R.M., Tabares, L., et al., 2013. Notch signaling pathway is activated in motoneurons of spinal muscular atrophy. Int. J. Mol. Sci. 14 (6), 11424-11437.

Chillakuri, C.R., Sheppard, D., Lea, S.M., Handford, P.A., 2012. Notch receptor-ligand binding and activation: insights from molecular studies. Semin. Cell Dev. Biol. 23 (4), 421-428.

de Bivort, B.L., Guo, H.F., Zhong, Y., 2009. Notch signaling is required for activity-dependent synaptic plasticity at the Drosophila neuromuscular junction. J. Neurogenet. 23 (4), 395-404.

Dearmond, S.J., Bajsarowicz, K., 2010. PrPSc accumulation in neuronal plasma membranes links Notch-1 activation to dendritic degeneration in prion diseases. Mol. Neurodegener. 5, 6 .

del Alamo, D., Rouault, H., Schweisguth, F., 2011. Mechanism and significance of cisinhibition in notch signalling. Curr. Biol. 21 (1), R40-R47.

D'Souza, B., Miyamoto, A., Weinmaster, G., 2008. The many facets of notch ligands. Oncogene 27 (38), 5148-5167.

d'Ydewalle, C., Krishnan, J., Chiheb, D.M., Van Damme, P., Irobi, J., Kozikowski, A.P., et al., 2011. HDAC6 inhibitors reverse axonal loss in a mouse model of mutant HSPB1-induced Charcot-Marie-tooth disease. Nat. Med. 17 (8), 968-974.

El Bejjani, R., Hammarlund, M., 2012. Notch signaling inhibits axon regeneration. Neuron 73 (2), 268-278.

Fior, R., Henrique, D., 2005. A novel hes5/hes6 circuitry of negative regulation controls notch activity during neurogenesis. Dev. Biol. 281 (2), 318-333.

Genoud, S., Lappe-Siefke, C., Goebbels, S., Radtke, F., Aguet, M., Scherer, S.S., et al., 2002. Notch1 control of oligodendrocyte differentiation in the spinal cord. J. Cell Biol. 158 (4), 709-718.

Grandbarbe, L., Michelucci, A., Heurtaux, T., Hemmer, K., Morga, E., Heuschling, P., 2007. Notch signaling modulates the activation of microglial cells. Glia 55 (15), 1519-1530.

Gurney, M.E., 1994. Transgenic-mouse model of amyotrophic lateral sclerosis. N. Engl. J. Med. 331 (25), 1721-1722.

Haidet-Phillips, A.M., Hester, M.E., Miranda, C.J., Meyer, K., Braun, L., Frakes, A., et al., 2011. Astrocytes from familial and sporadic ALS patients are toxic to motor neurons. Nat. Biotechnol. 29 (9), 824-828.

Hall, E.D., Oostveen, J.A., Gurney, M.E., 1998. Relationship of microglial and astrocytic activation to disease onset and progression in a transgenic model of familial ALS. Glia 23 (3), 249-256.

Hamada, Y., Kadokawa, Y., Okabe, M., Ikawa, M., Coleman, J.R., Tsujimoto, Y., 1999 Mutation in ankyrin repeats of the mouse Notch2 gene induces early embryonic lethality. Development 126 (15), 3415-3424.

Hansson, E.M., Lanner, F., Das, D., Mutvei, A., Marklund, U., Ericson, J., et al., 2010. Control of notch-ligand endocytosis by ligand-receptor interaction. J. Cell Sci. 123, 2931-2942 Pt 17.

Hatakeyama, J., Bessho, Y., Katoh, K., Ookawara, S., Fujioka, M., Guillemot, F., et al. 2004. Hes genes regulate size, shape and histogenesis of the nervous system by control of the timing of neural stem cell differentiation. Development 131 (22), 5539-5550.

He, W., Hu, J., Xia, Y., Yan, R., 2014. beta-site amyloid precursor protein cleaving enzyme 1(BACE1) regulates notch signaling by controlling the cleavage of Jagged 1 (Jag1) and Jagged 2 (Jag2). J. Biol. Chem. 289 (30), 20630-20637.

Henkel, J.S., Beers, D.R., Zhao, W., Appel, S.H., 2009. Microglia in ALS: the good, the bad, and the resting. J. NeuroImmune Pharmacol. 4 (4), 389-398.

Huppert, S.S., Le, A., Schroeter, E.H., Mumm, J.S., Saxena, M.T., Milner, L.A., et al., 2000. Embryonic lethality in mice homozygous for a processing-deficient allele of Notch1. Nature 405 (6789), 966-970.

Ilagan, M.X., Kopan, R., 2007. SnapShot: notch signaling pathway. Cell 128 (6), 1246.

Ilieva, H., Polymenidou, M., Cleveland, D.W., 2009. Non-cell autonomous toxicity in neurodegenerative disorders: ALS and beyond. J. Cell Biol. 187 (6), 761-772.

Ishikura, N., Clever, J.L., Bouzamondo-Bernstein, E., Samayoa, E., Prusiner, S.B., Huang, E.J., et al., 2005. Notch-1 activation and dendritic atrophy in prion disease. Proc. Natl. Acad. Sci. U. S. A. 102 (3), 886-891.

John, G.R., Shankar, S.L., Shafit-Zagardo, B., Massimi, A., Lee, S.C., Raine, C.S., et al., 2002. Multiple sclerosis: re-expression of a developmental pathway that restricts oligodendrocyte maturation. Nat. Med. 8 (10), 1115-1121.

Jurynczyk, M., Selmaj, K., 2010. Notch: a new player in MS mechanisms. J. Neuroimmunol. 218 (1-2), 3-11.

Kamei, N., Kwon, S.M., Ishikawa, M., Ii, M., Nakanishi, K., Yamada, K., et al., 2012. Endothelial progenitor cells promote astrogliosis following spinal cord injury through Jagged1-dependent notch signaling. J. Neurotrauma 29 (9), 1758-1769.

Kang, S.H., Li, Y., Fukaya, M., Lorenzini, I., Cleveland, D.W., Ostrow, L.W., et al., 2013. Degeneration and impaired regeneration of gray matter oligodendrocytes in amyotrophic lateral sclerosis. Nat. Neurosci. 16 (5), 571-579.

Kiernan, A.E., Xu, J., Gridley, T., 2006. The notch ligand JAG1 is required for sensory progenitor development in the mammalian inner ear. PLoS Genet. 2 (1), e4.

Kochert, K., Ullrich, K., Kreher, S., Aster, J.C., Kitagawa, M., Johrens, K., et al., 2011. High-level expression of mastermind-like 2 contributes to aberrant activation of the NOTCH signaling pathway in human lymphomas. Oncogene 30 (15), 1831-1840.

Kopan, R., Ilagan, M.X., 2009. The canonical notch signaling pathway: unfolding the activation mechanism. Cell 137 (2), 216-233.

Lai, E.C., 2004. Notch signaling: control of cell communication and cell fate. Development 131 (5), 965-973.

Larabee, J.L., Shakir, S.M., Barua, S., Ballard, J.D., 2013. Increased cAMP in monocytes 
augments notch signaling mechanisms by elevating RBP-J and transducin-like enhancer of split (TLE). J. Biol. Chem. 288 (30), 21526-21536.

Lavoie, M.J., Selkoe, D.J., 2003. The notch ligands, jagged and delta, are sequentially processed by alpha-secretase and presenilin/gamma-secretase and release signaling fragments. J. Biol. Chem. 278 (36), 34427-34437.

Le Bras, S., Loyer, N., Le Borgne, R., 2011. The multiple facets of ubiquitination in the regulation of notch signaling pathway. Traffic 12 (2), 149-161.

Lee, Y., Morrison, B.M., Li, Y., Lengacher, S., Farah, M.H., Hoffman, P.N., et al., 2012. Oligodendroglia metabolically support axons and contribute to neurodegeneration. Nature 487 (7408), 443-448.

Louvi, A., Artavanis-Tsakonas, S., 2006. Notch signalling in vertebrate neural development. Nat. Rev. Neurosci. 7 (2), 93-102.

Marumo, T., Takagi, Y., Muraki, K., Hashimoto, N., Miyamoto, S., Tanigaki, K., 2013. Notch signaling regulates nucleocytoplasmic Olig2 translocation in reactive astrocytes differentiation after ischemic stroke. Neurosci. Res. 75 (3), 204-209.

Mathieu, P., Adami, P.V., Morelli, L., 2013. Notch signaling in the pathologic adult brain. Biomol. Concepts 4 (5), 465-476.

Morrison, B.M., Lee, Y., Rothstein, J.D., 2013. Oligodendroglia: metabolic supporters of axons. Trends Cell Biol. 23 (12), 644-651.

Mukhopadhyay, A., Jarrett, J., Chlon, T., Kessler, J.A., 2009. HeyL regulates the number of TrkC neurons in dorsal root ganglia. Dev. Biol. 334 (1), 142-151.

Nave, K.A., 2010. Myelination and the trophic support of long axons. Nat. Rev. Neurosci. 11 (4), 275-283.

Nonneman, A., Robberecht, W., Van Den Bosch, L., 2014. The role of oligodendroglial dysfunction in amyotrophic lateral sclerosis. Neurodegener. Dis. Manag. 4 (3), 223-239.

Oka, C., Nakano, T., Wakeham, A., de la Pompa, J.L., Mori, C., Sakai, T., et al., 1995. Disruption of the mouse RBP-J kappa gene results in early embryonic death. Development 121 (10), 3291-3301.

Park, H.C., Appel, B., 2003. Delta-notch signaling regulates oligodendrocyte specification. Development 130 (16), 3747-3755.

Petrovic, J., Galvez, H., Neves, J., Abello, G., Giraldez, F., 2015. Differential regulation of Hes/Hey genes during inner ear development. Dev. Neurobiol. 75 (7), 703-720.

Philips, T., Robberecht, W., 2011. Neuroinflammation in amyotrophic lateral sclerosis: role of glial activation in motor neuron disease. Lancet Neurol. 10 (3), 253-263.

Philips, T., Bento-Abreu, A., Nonneman, A., Haeck, W., Staats, K., Geelen, V., et al., 2013. Oligodendrocyte dysfunction in the pathogenesis of amyotrophic lateral sclerosis. Brain 136 (Pt 2), 471-482.

Popko, B., 2003. Notch signaling: a rheostat regulating oligodendrocyte differentiation? Dev. Cell 5 (5), 668-669.

Rabin, S.J., Kim, J.M., Baughn, M., Libby, R.T., Kim, Y.J., Fan, Y., et al., 2010. Sporadic ALS has compartment-specific aberrant exon splicing and altered cell-matrix adhesion biology. Hum. Mol. Genet. 19 (2), 313-328.

Redmond, L., Oh, S.R., Hicks, C., Weinmaster, G., Ghosh, A., 2000. Nuclear Notch1 signaling and the regulation of dendritic development. Nat. Neurosci. 3 (1), 30-40.

Renton, A.E., Chio, A., Traynor, B.J., 2014. State of play in amyotrophic lateral sclerosis genetics. Nat. Neurosci. 17 (1), 17-23.

Sato, C., Zhao, G., Ilagan, M.X., 2012. An overview of notch signaling in adult tissue renewal and maintenance. Curr. Alzheimer Res. 9 (2), 227-240.

Sestan, N., Artavanis-Tsakonas, S., Rakic, P., 1999. Contact-dependent inhibition of cortical neurite growth mediated by notch signaling. Science 286 (5440), 741-746.

Shimada, I.S., Borders, A., Aronshtam, A., Spees, J.L., 2011. Proliferating reactive astrocytes are regulated by Notch-1 in the peri-infarct area after stroke. Stroke 42 (11), $3231-3237$.

Slezak, M., Goritz, C., Niemiec, A., Frisen, J., Chambon, P., Metzger, D., et al., 2007. Transgenic mice for conditional gene manipulation in astroglial cells. Glia 55 (15), $1565-1576$.

Staats, K.A., Humblet-Baron, S., Bento-Abreu, A., Scheveneels, W., Nikolaou, A., Deckers, K., et al., 2016. Genetic ablation of IP3 receptor 2 increases cytokines and decreases survival of SOD1G93A mice. Hum. Mol. Genet. 25 (16), 3491-3499.

Swinnen, B., Robberecht, W., 2014. The phenotypic variability of amyotrophic lateral sclerosis. Nat. Rev. Neurol. 10 (11), 661-670.

Van Damme, P., Robberecht, W., Van Den Bosch, L., 2017. Modelling amyotrophic lateral sclerosis: progress and possibilities. Dis. Model. Mech. 10 (5), 537-549.

von Grabowiecki, Y., Licona, C., Palamiuc, L., Abreu, P., Vidimar, V., Coowar, D., et al., 2015. Regulation of a Notch3-Hes1 pathway and protective effect by a tocopherolomega alkanol chain derivative in muscle atrophy. J. Pharmacol. Exp. Ther. 352 (1), 23-32.

Wang, S., Sdrulla, A.D., Disibio, G., Bush, G., Nofziger, D., Hicks, C., et al., 1998. Notch receptor activation inhibits oligodendrocyte differentiation. Neuron 21 (1), 63-75.

Wei, Z., Chigurupati, S., Arumugam, T.V., Jo, D.G., Li, H., Chan, S.L., 2011. Notch activation enhances the microglia-mediated inflammatory response associated with focal cerebral ischemia. Stroke 42 (9), 2589-2594.

Woo, H.N., Park, J.S., Gwon, A.R., Arumugam, T.V., Jo, D.G., 2009. Alzheimer's disease and notch signaling. Biochem. Biophys. Res. Commun. 390 (4), 1093-1097.

Yamanaka, K., Komine, O., 2018. The multi-dimensional roles of astrocytes in ALS. Neurosci. Res. 126, 31-38.

Yamanaka, K., Chun, S.J., Boillee, S., Fujimori-Tonou, N., Yamashita, H., Gutmann, D.H., et al., 2008. Astrocytes as determinants of disease progression in inherited amyotrophic lateral sclerosis. Nat. Neurosci. 11 (3), 251-253.

Yang, X., Klein, R., Tian, X., Cheng, H.T., Kopan, R., Shen, J., 2004. Notch activation induces apoptosis in neural progenitor cells through a p53-dependent pathway. Dev. Biol. 269 (1), 81-94.

Yang, D., Abdallah, A., Li, Z., Lu, Y., Almeida, S., Gao, F.B., 2015. FTD/ALS-associated poly(GR) protein impairs the notch pathway and is recruited by poly(GA) into cytoplasmic inclusions. Acta Neuropathol. 130 (4), 525-535.

Yoon, K., Gaiano, N., 2005. Notch signaling in the mammalian central nervous system: insights from mouse mutants. Nat. Neurosci. 8 (6), 709-715.

Zhan, L., Hanson, K.A., Kim, S.H., Tare, A., Tibbetts, R.S., 2013. Identification of genetic modifiers of TDP-43 neurotoxicity in Drosophila. PLoS ONE 8 (2), e57214.

Zhang, Y., Chen, K.N., Sloan, S.A., Bennett, M.L., Scholze, A.R., O'Keeffe, S., et al., 2014 An RNA-sequencing transcriptome and splicing database of glia, neurons, and vascular cells of the cerebral cortex. J. Neurosci. 34 (36), 11929-11947.

Zhang, Y., He, K., Wang, F., Li, X., Liu, D., 2015. Notch-1 signaling regulates astrocytic proliferation and activation after hypoxia exposure. Neurosci. Lett. 603, 12-18. 